\title{
Wave propagation in a microstretch thermoelastic diffusion solid
}

\author{
Rajneesh Kumar
}

\begin{abstract}
The present article deals with the two parts: (i)The propagation of plane waves in a microstretch thermoelastic diffusion solid of infinite extent. (ii)The reflection and transmission of plane waves at a plane interface between inviscid fluid half-space and micropolar thermoelastic diffusion solid half-space.It is found that for two-dimensional model, there exist four coupled longitudinal waves, that is, longitudinal displacement wave (LD), thermal wave (T), mass diffusion wave (MD) and longitudinal microstretch wave (LM) and two coupled transverse waves namely (CD-I and CD-II waves). The phase velocity, attenuation coefficient, specific loss and penetration depth are computed numerically and depicted graphically. In the second part, it is noticed that the amplitude ratios of various reflected and transmitted waves are functions of angle of incidence, frequency of incident wave and are influenced by the microstretch thermoelastic diffusion properties of the media. The expressions of amplitude ratios and energy ratios are obtained in closed form. The energy ratios have been computed numerically for a particular model. The variations of energy ratios with angle of incidence for thermoelastic diffusion media in the context of Lord-Shulman (L-S) [1] and Green-Lindsay (G-L) [2] theories are depicted graphically. Some particular cases are also deduced from the present investigation.
\end{abstract}

Key Words: Microstretch, Phase velocity, Attenuation coefficient, Specific loss, Penetration depth, Reflection, Transmission, Energy ratios.

Received: 28 April, 2014.

Revised: 20 June, 2014.

Accepted: 28 June, 2014. 


\section{Introduction}

Eringen $[3,4]$ developed the theory of micromorphic bodies. The theory of microstretch elastic solids developed by Eringen [5] is a generalization of the micropolar theory. Eringen [6] also developed the theory of thermomicrostretch elastic solids. The particles of microstretch materials have seven degree of freedom: three for displacements, three for microrotations and one for microstretch. A microstretch continuum can be model as composite materials reinforced with chopped elastic fibres and various porous solids. The material points of these bodies can stretch and contract independently of their translations and rotations. A book by Eringen [7] gave an exposition of the development in the microcontinuum field theories for solids (micromorphic, microstretch, and microplar) including electromagnetic and thermal interactions.

Various investigators have studied different types of problems in microstretch thermoelastic medium notable among them are Ciarletta and Scalia [8], Iesan and Quintanilla [9], Othman et al [10], Passarella and Tibullo [11], Marin [12, 13], Kumar et al [14], Othman and Lofty [15, 16], Kumar and Rupender [17, 18 .

Singh [19] studied the reflection and refraction of plane waves at a liquid/ thermo-microstretch elastic solid interface. Kumar and Pratap [20] discussed the reflection of plane waves in a heat flux dependent microstretch thermoelastic solid half space. Propagation of Rayleigh surface waves in microstretch thermoelastic continua under inviscid fluid loading have been investigated by Sharma et al. [21]. The propagation of free vibrations in microstretch thermoelastic homogeneous, isotropic, thermally conducting plate bordered with layers of inviscid liquid on both sides subjected to stress free thermally insulated and isothermal conditions have been investigated by Kumar and Pratap [22].

Diffusion is defined as the spontaneous movement of the particles from a high concentration region to the low concentration region and it occurs in response to a concentration gradient expressed as the change in the concentration due to change in position. Thermal diffusion utilizes the transfer of heat across a thin liquid or gas to accomplish isotope separation. Today, thermal diffusion remains a practical process to separate isotopes of noble gases(e.g. xexon) and other light isotopes(e.g. carbon) for research purposes. In most of the applications, the concentration is calculated using what is known as Fick's law. This is a simple law which does not take into consideration the mutual interaction between the introduced substance and the medium into which it is introduced or the effect of temperature on this interaction. However, there is a certain degree of coupling with temperature and temperature gradients as 
temperature speeds up the diffusion process. The thermodiffusion in elastic solids is due to coupling of fields of temperature, mass diffusion and that of strain in addition to heat and mass exchange with the environment.

Nowacki [23-26] developed the theory of thermoelastic diffusion by using coupled thermoelastic model. Dudziak and Kowalski [27] and Olesiak and Pyryev [28], respectively, discussed the theory of thermodiffusion and coupled quasi-stationary problems of thermal diffusion for an elastic layer. They studied the influence of cross effects arising from the coupling of the fields of temperature, mass diffusion and strain due to which the thermal excitation results in additional mass concentration and that generates additional fields of temperature.

Gawinecki and Szymaniec [29] proved a theorem about global existence of the solution for a non-linear parabolic thermoelastic diffusion. problem. Uniqueness and reciprocity theorems for the equations of generalized thermoelastic diffusion problem, in isotropic media, was proved by Sherief et al. [30] on the basis of the variational principle equations, under restrictive assumptions on the elastic coefficients. Due to the inherit complexity of the derivation of the variational principle equations, Aouadi [31] proved this theorem in the Laplace transform domain, under the assumption that the functions of the problem are continuous and the inverse Laplace transform of each is also unique. Sherief and Saleh [32] investigated the problem of a thermoelastic half-space in the context of the theory of generalized thermoelastic diffusion with one relaxation time. Miglani and Kaushal [33] studied the propagation of transverse and microrotational waves in micropolar generalized thermodiffusion elastic half space. Kumar and Kansal [34] developed the basic equation of anisotropic thermoelastic diffusion based upon Green-Lindsay model. Kumar and Kansal [35] investigated the fundamental solution in thermomicrostretch elastic diffusive solids.

The Propagation of plane waves at the interface of an elastic solid halfspace and a microstretch thermoelastic diffusion solid half-space was studied by Kumar, Garg and Ahuja [36]. They also discussed the Rayleigh wave propagation in isotropic microstrech thermoelastic diffusion solid half- space [37].

For the boundary value problem considered in the context of dipolar bodies with stretch, in the paper [41], the authors use some results from the theory of semigroups of the linear operators in order to prove the existence and uniqueness of a weak solution, for the initial boundary value problem of a porous thermoelastic body, the authors analyze the temporal behaviour of the solutions [43]. In the paper [42] the problem of reflection and transmission of plane waves at an imperfect boundary between two thermally conducting micropolar elastic solid half spaces with two temperature is investigated. In 
the paper [44] the authors establish some existence results of the problem at resonance under some appropriate conditions.

In the first part of the present paper, the propagation of plane waves in an microstretch generalized thermoelastic diffusion solid have been investigated. The phase velocity, attenuation coefficient, specific loss and penetration depth have been computed numerically and depicted graphically. In the second part, the reflection and refraction phenomenon at a plane interface between an inviscid fluid medium and a microstretch thermoelastic diffusion solid medium has been analyzed. In microstretch thermoelastic diffusion solid medium, potential functions are introduced to the equations. The amplitude ratios of various reflected and transmitted waves to that of incident wave are derived. These amplitude ratios are further used to find the expressions of energy ratios of various reflected and refracted waves to that of incident wave. The graphical representation is given for these energy ratios for different direction of propagation. The law of conservation of energy at the interface is verified.

\section{Basic equations}

Following Eringen [7], Sherief et al. [30] and Kumar and Kansal [34], the equations of motion and the constitutive relations in a homogeneous isotropic microstretch thermoelastic diffusion solid in the absence of body forces, body couples, stretch force, and heat sources are given by

$$
\begin{gathered}
(\lambda+2 \mu+K) \nabla(\nabla \cdot \vec{u})-(\mu+K) \nabla \times \nabla \times \vec{u}+K \nabla \times \vec{\varphi}+\lambda_{0} \nabla \varphi^{*} \\
-\beta_{1}\left(1+\tau_{1} \frac{\partial}{\partial t}\right) \nabla T-\beta_{2}\left(1+\tau^{1} \frac{\partial}{\partial t}\right) \nabla C=\rho \frac{\partial^{2} \vec{u}}{\partial t^{2}} \\
(\alpha+\beta+\gamma) \nabla(\nabla \cdot \vec{\varphi})-\gamma \nabla \times \nabla \times \vec{\varphi}+K \nabla \times \vec{u}-2 K \vec{\varphi}=\rho j \frac{\partial^{2} \vec{\varphi}}{\partial t^{2}}, \\
\alpha_{0} \nabla^{2} \varphi^{*}+\nu_{1}\left(1+\tau_{1} \frac{\partial}{\partial t}\right) T+\nu_{2}\left(1+\tau^{1} \frac{\partial}{\partial t}\right) C-\lambda_{1} \varphi^{*}-\lambda_{0} \nabla \cdot \vec{u}=\frac{\rho j_{0}}{2} \frac{\partial^{2} \varphi^{*}}{\partial t^{2}}, \\
K^{*} \nabla^{2} T=\beta_{1} T_{0}\left(1+\varepsilon \tau_{0} \frac{\partial}{\partial t}\right) \nabla \cdot \dot{\vec{u}}+\nu_{1} T_{0}\left(1+\varepsilon \tau_{0} \frac{\partial}{\partial t}\right) \dot{\varphi}^{*} \\
+\rho C^{*}\left(1+\tau_{0} \frac{\partial}{\partial t}\right) \dot{T}+a T_{0}\left(1+\gamma_{1} \frac{\partial}{\partial t}\right) \frac{\partial C}{\partial t}, \\
D \beta_{2} e_{k k, i i}+D \nu_{2} \varphi_{, i i}^{*}+D a\left(1+\tau_{1} \frac{\partial}{\partial t}\right) T_{, i i}+\left(1+\varepsilon \tau^{0} \frac{\partial}{\partial t}\right) \frac{\partial C}{\partial t}-D b\left(1+\tau^{1} \frac{\partial}{\partial t}\right) C_{, i i}=0
\end{gathered}
$$


and constitutive equations are

$$
\begin{gathered}
t_{i j}=\lambda u_{r, r} \delta_{i j}+\mu\left(u_{i, j}+u_{j, i}\right)+K\left(u_{j, i}-e_{i j r} \varphi_{r}\right)+\lambda_{0} \delta_{i j} \varphi^{*} \\
-\beta_{1}\left(1+\tau_{1} \frac{\partial}{\partial t}\right) T \delta_{i j}-\beta_{2}\left(1+\tau^{1} \frac{\partial}{\partial t}\right) C \delta_{i j}, \\
m_{i j}=\alpha \varphi_{r, r} \delta_{i j}+\beta \varphi_{i, j}+\gamma \varphi_{j, i}+b_{0} e_{m j i} \varphi_{, m}^{*}, \\
\lambda_{i}^{*}=\alpha_{0} \varphi_{, i}^{*}+b_{0} e_{i j m} \varphi_{j, m},
\end{gathered}
$$

where $\lambda, \mu, \alpha, \beta, \gamma, K, \lambda_{0}, \lambda_{1}, \alpha_{0}, b_{0}$, are material constants, $\rho$ is the mass density, $\vec{u}=\left(u_{1}, u_{2}, u_{3}\right)$ is the displacement vector and $\vec{\varphi}=\left(\varphi_{1}, \varphi_{2}, \varphi_{3}\right)$ is the microrotation vector, $\varphi^{*}$ is the microstretch scalar function, $T$ and $T_{0}$ are the small temperature increment and the reference temperature of the body chosen such that ||$T / T_{0} \mid<<1, C$ is the concentration of the diffusion material in the elastic body, $K^{*}$ is the coefficient of the thermal conductivity, $C^{*}$ is the specific heat at constant strain; $D$ is the thermoelatic diffusion constant, $a, b$ are, respectively, coefficients describing the measure of thermoelastic diffusion effects and of mass diffusion effects, $\beta_{1}=(3 \lambda+2 \mu+K) \alpha_{t 1}$, $\beta_{2}=(3 \lambda+2 \mu+K) \alpha_{c 1}, \nu_{1}=(3 \lambda+2 \mu+K) \alpha_{t 2}, \nu_{2}=(3 \lambda+2 \mu+K) \alpha_{c 2} ;$ $\alpha_{t 1}, \alpha_{t 2}$ are coefficients of linear thermal expansion and $\alpha_{c 1}, \alpha_{c 2}$ are coefficients of linear diffusion expansion, $j$ is the microinertia, $j_{0}$ is the microinertia of the microelements, $\sigma_{i j}$ and $m_{i j}$ are components of stress and couple stress tensors respectively, $\lambda_{i}^{*}$ is the microstress tensor,$e_{i j}=\left(u_{i, j}+u_{j, i}\right) / 2$ are components of infinitesimal strain, $e_{k k}$ is the dilatation, $\delta_{i j}$ is the Kronecker delta, $\tau^{0}, \tau^{1}$ are diffusion relaxation times with $\tau^{1} \geq \tau^{0} \geq 0$ and $\tau_{0}, \tau_{1}$ are thermal relaxation times with $\tau_{1} \geq \tau_{0} \geq 0$. Here $\tau_{0}=\tau^{0}=\tau_{1}=\tau^{1}=0$ for Coupled Thermoelasticity $(\mathrm{CT})$ model, $\tau_{1}=\tau^{1}=0, \varepsilon=1, \gamma_{1}=\tau_{0}$ for Lord-Shulman(L-S) model and $\varepsilon=0, \gamma_{1}=\tau^{0}$ for Green-Lindsay(G-L) model.

In the above equations, a comma followed by a suffix denotes spatial derivative and a superposed dot denotes the derivative with respect to time respectively. For two-dimensional problem, we have

$$
\vec{u}=\left(u_{1}, u_{2}, u_{3}\right), \vec{\varphi}=\left(0, \varphi_{2}, 0\right) .
$$

We define the following dimensionless quantities

$$
\begin{gathered}
\left(x_{1}^{\prime}, x_{3}^{\prime}\right)=\frac{w^{*}}{c_{1}}\left(x_{1}, x_{3}\right),\left(u_{1}^{\prime}, u_{3}^{\prime}\right)=\frac{\rho c_{1} w^{*}}{\beta_{1} T_{0}}\left(u_{1}, u_{3}\right), t_{i j}^{\prime}=\frac{t_{i j}^{\prime}}{\beta_{1} T_{0}}, t^{\prime}=w^{*} t, \tau_{0}^{\prime}=w^{*} \tau_{0}, \\
\tau_{1}^{\prime}=w^{*} \tau_{1}, \tau^{0^{\prime}}=w^{*} \tau^{0}, \tau^{1^{\prime}}=w^{*} \tau^{1}, T^{\prime}=\frac{T}{T_{0}}, C^{\prime}=\frac{\beta_{2} C}{\rho c_{1}^{2}},\left(u_{1}^{f^{\prime}}, u_{3}^{f^{\prime}}\right)=\frac{\rho c_{1} w^{*}}{\beta_{1} T_{0}}\left(u_{1}^{f}, u_{3}^{f}\right),
\end{gathered}
$$




$$
\begin{gathered}
\varphi^{*^{\prime}}=\frac{\rho c_{1}^{2}}{\beta_{1} T_{0}} \varphi^{*}, \lambda_{i}^{*^{\prime}}=\frac{w^{*}}{c_{1} \beta_{1} T_{0}} \lambda_{i}^{*}, \varphi_{2^{\prime}}=\frac{\rho c_{1}^{2}}{\beta_{1} T_{0}} \varphi_{2}, \varphi^{f^{\prime}}=\frac{w^{*}}{c_{1}^{2}} \varphi^{f}, p^{f^{\prime}}=\frac{1}{\beta_{1} T_{0}} p^{f}, \\
m_{i j}^{\prime}=\frac{w^{*}}{c_{1} \beta_{1} T_{0}} m_{i j}, P_{i j}^{*^{\prime}}=\frac{\rho c_{1}}{\beta_{1}^{2} T_{0}^{2}} P_{i j}^{*}, P^{* f^{\prime}}=\frac{\rho c_{1}}{\beta_{1}^{2} T_{0}^{2}} P^{* f} .
\end{gathered}
$$

where $w^{*}=\frac{\rho C^{*} c_{1}^{2}}{K^{*}}, c_{1}^{2}=\frac{\lambda+2 \mu+K}{\rho}, w^{*}$ is the characteristic frequency of the medium.

Upon introducing the quantities (1.10) in equations (1.1)-(1.5), with the aid of (1.9) and after suppressing the primes, we obtain

$$
\begin{gathered}
\delta^{2} \frac{\partial e}{\partial x_{1}}+\left(1-\delta^{2}\right) \nabla^{2} u_{1}-\zeta_{1}^{*} \frac{\partial \varphi_{2}}{\partial x_{3}}+\zeta_{3}^{*} \frac{\partial \varphi^{*}}{\partial x_{1}}-\tau_{t}^{1} \frac{\partial T}{\partial x_{1}}-\zeta_{2}^{*} \tau_{c}^{1} \frac{\partial C}{\partial x_{1}}=\frac{\partial^{2} u_{1}}{\partial t^{2}} \\
\delta^{2} \frac{\partial e}{\partial x_{3}}+\left(1-\delta^{2}\right) \nabla^{2} u_{3}+\zeta_{1}^{*} \frac{\partial \varphi_{2}}{\partial x_{1}}+\zeta_{3}^{*} \frac{\partial \varphi^{*}}{\partial x_{3}}-\tau_{t}^{1} \frac{\partial T}{\partial x_{3}}-\zeta_{2}^{*} \tau_{c}^{1} \frac{\partial C}{\partial x_{3}}=\frac{\partial^{2} u_{3}}{\partial t^{2}} \\
\zeta_{1} \nabla^{2} \varphi_{2}+\zeta_{2}\left(\frac{\partial u_{1}}{\partial x_{3}}-\frac{\partial u_{3}}{\partial x_{1}}\right)-\zeta_{3} \varphi_{2}=\frac{\partial^{2} \varphi_{2}}{\partial t^{2}} \\
\left(\delta_{1}^{2} \nabla^{2}-\chi_{1}^{*}\right) \varphi^{*}-\chi_{2}^{*} e+\chi_{3}^{*} \tau_{t}^{1} T+\chi_{4}^{*} \tau_{c}^{1} C=\frac{\partial^{2} \varphi^{*}}{\partial t^{2}} \\
\nabla^{2} T=l_{1}^{*} \tau_{e}^{0} \frac{\partial e}{\partial t}+l_{2}^{*} \tau_{e}^{0} \frac{\partial \varphi^{*}}{\partial t}+\tau_{t}^{0} \frac{\partial T}{\partial t}+l_{3}^{*} \tau_{c}^{0} \frac{\partial C}{\partial t} \\
q_{1}^{*} \nabla^{2} e+q_{4}^{*} \nabla^{2} \varphi^{*}+q_{2}^{*} \tau_{t}^{1} \nabla^{2} T+\tau_{f}^{0} \frac{\partial C}{\partial t}-q_{3}^{*} \tau_{c}^{1} \nabla^{2} C=0
\end{gathered}
$$

where,

$$
\begin{gathered}
\zeta_{1}=\frac{\gamma}{j \rho c_{1}^{2}}, \zeta_{2}=\frac{K}{j \rho w^{* 2}}, \zeta_{3}=\frac{2 K}{j \rho w^{* 2}}, \zeta_{1}^{*}=\frac{K}{\rho c_{1}^{2}}, \zeta_{2}^{*}=\frac{\rho c_{1}^{2}}{\beta_{1} T_{0}}, \zeta_{3}^{*}=\frac{\lambda_{0}}{\rho c_{1}^{2}}, \\
\delta^{2}=\frac{\lambda+\mu}{\rho c_{1}^{2}}, l_{1}^{*}=\frac{T_{0} \beta_{1}^{2}}{\rho K^{*} w^{*}}, l_{2}^{*}=\frac{T_{0} \beta_{1} \nu_{1}}{\rho K^{*} w^{*}}, l_{3}^{*}=\frac{\rho c_{1}^{4} a}{\beta_{2} K^{*} w^{*}}, q_{1}^{*}=\frac{D w^{*} \beta_{1}^{2}}{\rho c_{1}^{4}}, q_{2}^{*}=\frac{D w^{*} \beta_{2} a}{\beta_{1} c_{1}^{2}}, \\
q_{3}^{*}=\frac{D b w^{*}}{c_{1}^{2}}, q_{4}^{*}=\frac{D \nu_{2} \beta_{2} w^{*}}{\rho c_{1}^{4}}, \chi_{1}^{*}=\frac{2 \lambda}{\rho j_{0} w^{* 2}}, \chi_{2}^{*}=\frac{2 \lambda_{0}}{\rho j_{0} w^{* 2}}, \chi_{3}^{*}=\frac{2 \nu_{1} c_{1}^{2}}{j_{0} \beta_{1} w^{* 2}}, \chi_{4}^{*}=\frac{2 \nu_{2} \rho c_{1}^{4}}{j_{0} \beta_{1} \beta_{2} T_{0} w^{* 2}}, \\
\delta_{1}^{2}=\frac{c_{2}^{2}}{c_{1}^{2}}, c_{2}^{2}=\frac{2 \alpha_{0}}{\rho j_{0}}, \tau_{t}^{1}=1+\tau_{1} \frac{\partial}{\partial t}, \tau_{c}^{1}=1+\tau^{1} \frac{\partial}{\partial t}, \tau_{f}^{0}=1+\varepsilon \tau^{0} \frac{\partial}{\partial t}, \tau_{t}^{0}=1+\tau_{0} \frac{\partial}{\partial t}, \\
\tau_{e}^{0}=1+\varepsilon \tau_{0} \frac{\partial}{\partial t}, \tau_{c}^{0}=1+\gamma_{1} \frac{\partial}{\partial t}, e=\frac{\partial u_{1}}{\partial x_{1}}+\frac{\partial u_{3}}{\partial x_{3}}, \nabla^{2}=\frac{\partial^{2}}{\partial x_{1}^{2}}+\frac{\partial^{2}}{\partial x_{3}^{2}} .
\end{gathered}
$$

Introducing the potential functions $\phi$ and $\psi$ through the relations

$$
u_{1}=\frac{\partial \phi}{\partial x_{1}}-\frac{\partial \psi}{\partial x_{3}}, u_{3}=\frac{\partial \phi}{\partial x_{3}}+\frac{\partial \psi}{\partial x_{1}},
$$


in the equations $(1.11)-(1.16)$, we obtain

$$
\begin{gathered}
\nabla^{2} \phi+\zeta_{3}^{*} \varphi^{*}-\tau_{t}^{1} T-\zeta_{2}^{*} \tau_{c}^{1} C=\frac{\partial^{2} \phi}{\partial t^{2}} \\
\left(1-\delta^{2}\right) \nabla^{2} \psi+\zeta_{1}^{*} \varphi_{2}=\frac{\partial^{2} \psi}{\partial t^{2}} \\
\left(\zeta_{1} \nabla^{2}-\zeta_{3}\right) \varphi_{2}-\zeta_{2} \nabla^{2} \psi=\frac{\partial^{2} \varphi_{2}}{\partial t^{2}}, \\
\left(\delta_{1}^{2} \nabla^{2}-\chi_{1}^{*}\right) \varphi^{*}-\chi_{2}^{*} \nabla^{2} \phi+\chi_{3}^{*} \tau_{t}^{1} T+\chi_{4}^{*} \tau_{c}^{1} C=\frac{\partial^{2} \varphi^{*}}{\partial t^{2}}, \\
\nabla^{2} T=\tau_{e}^{0} \frac{\partial}{\partial t}\left(l_{1}^{*} \nabla^{2} \phi+l_{2}^{*} \varphi^{*}\right)+\tau_{t}^{0} \frac{\partial T}{\partial t}+l_{3}^{*} \tau_{c}^{0} \frac{\partial C}{\partial t} \\
q_{1}^{*} \nabla^{4} \phi+q_{4}^{*} \nabla^{2} \varphi^{*}+q_{2}^{*} \tau_{t}^{1} \nabla^{2} T+\tau_{f}^{0} \frac{\partial C}{\partial t}-q_{3}^{*} \tau_{c}^{1} \nabla^{2} C=0 .
\end{gathered}
$$

\section{Plane wave propagation:}

For plane harmonic waves, we assume the solution of the form

$$
\left(\phi, \psi, T, C, \varphi^{*}, \varphi_{2}\right)=\left(\bar{\phi}, \bar{\psi}, \bar{T}, \bar{C}, \overline{\varphi^{*}}, \overline{\varphi_{2}}\right) \exp \left[\iota\left(\left(x_{1} l_{1}+x_{3} l_{3}\right)-\omega t\right)\right],
$$

where $\omega$ is the angular frequency. $\bar{\phi}, \bar{\psi}, \bar{T}, \bar{C}, \overline{\varphi^{*}}, \overline{\varphi_{2}}$ are undetermined amplitude vectors that are independent of time $t$ and coordinates $x_{m}(m=1,3) . l_{1}$ and $l_{3}$ are the direction cosines of the wave normal to the $x_{1} x_{3^{-}}$plane with the property $l_{1}^{2}+l_{3}^{2}=1$.

Substituting the values of $\phi, \psi, T, C, \varphi^{*}, \varphi_{2}$ from equation (2.1) in the equations $(1.18)-(1.23)$, we obtain

$$
\begin{gathered}
\left(w^{2}-\xi^{2}\right) \bar{\phi}+\zeta_{3}^{*} \bar{\varphi}^{*}-\tau_{t}^{11} \bar{T}-\zeta_{2}^{*} \tau_{c}^{11} \bar{C}=0 \\
\left(w^{2}-\left(1-\delta^{2}\right) \xi^{2}\right) \bar{\psi}+\zeta_{1}^{*} \bar{\varphi}_{2}=0 \\
-\xi^{2} \zeta_{2} \bar{\psi}+\left(-w^{2}+\zeta_{1} \xi^{2}+\zeta_{3}\right) \bar{\varphi}_{2}=0 \\
\chi_{2}^{*} \xi^{2} \bar{\phi}+\chi_{3}^{*} \tau_{t}^{11} \bar{T}+\left(w^{2}-\delta_{1}^{2} \xi^{2}-\chi_{1}^{*}\right) \bar{\varphi}^{*}+\chi_{4}^{*}(1-\iota w) \bar{C}=0, \\
-l_{1}^{*} \tau_{e}^{10} \xi^{2} \bar{\phi}+\left(\tau_{t}^{10}+\xi^{2}\right) \bar{T}+l_{2}^{*} \tau_{e}^{10} \bar{\varphi}^{*}+l_{3}^{*} \tau_{c}^{10} \bar{C}=0,
\end{gathered}
$$




$$
q_{1}^{*} \xi^{4} \bar{\phi}-q_{4}^{*} \xi^{2} \bar{\varphi}^{*}-q_{2}^{*} \tau_{t}^{11} \xi^{2} \bar{T}+\left(\tau_{f}^{10}+q_{3}^{*} \tau_{c}^{11} \xi^{2}\right) \bar{C}=0
$$

where

$$
\begin{gathered}
\tau_{t}^{11}=1-\iota \omega \tau_{1}, \tau_{c}^{11}=1-\iota \omega \tau^{1}, \tau_{t}^{10}=-\iota w\left(1-\iota \omega \tau_{0}\right), \tau_{c}^{10}=-\iota w\left(1-\iota \omega \gamma_{1}\right), \\
\tau_{e}^{10}=-\iota w\left(1-\iota \omega \varepsilon \tau_{0}\right), \tau_{f}^{10}=-\iota w\left(1-\iota \omega \varepsilon \tau^{0}\right) .
\end{gathered}
$$

The system of equations $(2.2),(2.5)-(2.7)$ has a non-trivial solution if the determinant of the coefficients $\left[\bar{\phi}, \bar{T}, \bar{\varphi}^{*}, \bar{C}\right]^{T}$ vanishes, which yields to the following polynomial characteristic equation in $\xi$ as:

$$
G_{1} \xi^{8}+G_{2} \xi^{6}+G_{3} \xi^{4}+G_{4} \xi^{2}+G_{5}=0
$$

where,

$$
\begin{gathered}
G_{1}=M_{1}-M_{11} F_{13}, G_{2}=w^{2} M_{1}+M_{2}-F_{4} M_{5}+F_{9} M_{8}-F_{13} M_{12}, \\
G_{3}=w^{2} M_{2}+M_{3}-F_{4} M_{6}+F_{9} M_{9}-F_{13} M_{13}, \\
G_{4}=w^{2} M_{3}+M_{4}-F_{4} M_{7}+F_{9} M_{10}, \\
G_{5}=w^{2} M_{4}
\end{gathered}
$$

and

$$
\begin{gathered}
M_{1}=F_{6} F_{17}, M_{2}=-F_{6} F_{10} F_{17}+F_{7} F_{17}+F_{6} F_{16}-F_{8} F_{14}+F_{6} F_{12} F_{15}, \\
M_{3}=F_{5}\left(F_{11} F_{17}-F_{12} F_{14}\right)-F_{10}\left(F_{7} F_{17}+F_{6} F_{16}-F_{8} F_{14}\right)+F_{7} F_{16}+F_{15}\left(F_{7} F_{12}-F_{8} F_{11}\right), \\
M_{4}=F_{16}\left(F_{5} F_{11}-F_{7} F_{10}\right), M_{5}=\left(F_{2} F_{17}-F_{3} F_{14}\right), \\
M_{6}=F_{2}\left(F_{11} F_{17}-F_{12} F_{14}\right)-F_{10}\left(F_{1} F_{17}-F_{3} F_{14}\right)+F_{1} F_{16}+F_{15}\left(F_{1} F_{12}-F_{3} F_{11}\right), \\
M_{7}=F_{16}\left(F_{2} F_{11}-F_{1} F_{10}\right), M_{8}=F_{6}\left(F_{2} F_{17}-F_{3} F_{15}\right), \\
M_{9}=\left(F_{7} F_{17}+F_{6} F_{16}-F_{8} F_{14}\right)-F_{5}\left(F_{1} F_{17}-F_{3} F_{14}\right)+F_{15}\left(F_{1} F_{8}-F_{3} F_{7}\right), \\
M_{10}=F_{16}\left(F_{2} F_{7}-F_{1} F_{5}\right), M_{11}=-F_{3} F_{6}, M_{12}=\left(F_{2} F_{6} F_{12}+F_{3} F_{6} F_{10}-F_{1} F_{8}+F_{3} F_{7}\right), \\
M_{13}=F_{2}\left(F_{7} F_{12}-F_{8} F_{11}\right)-F_{5}\left(F_{1} F_{12}-F_{3} F_{11}\right)+F_{10}\left(F_{1} F_{8}-F_{3} F_{7}\right), \\
F_{1}=\zeta_{3}^{*}, F_{2}=-\tau_{t}^{11}, F_{3}=-\zeta_{2}^{*} \tau_{c}^{11}, F_{4}=-\chi_{2}^{*}, F_{5}=\chi_{3}^{*} \tau_{t}^{11}, F_{6}=\delta_{1}^{2}, F_{7}=w^{2}-\chi_{1}^{*}, \\
F_{8}=\chi_{4}^{*}(1-\iota w), F_{9}=l_{1}^{*} \tau_{e}^{10}, F_{10}=\tau_{t}^{10}, F_{11}=l_{2}^{*} \tau_{e}^{10}, F_{12}=l_{3}^{*} \tau_{c}^{10}, F_{13}=q_{1}^{*}, F_{14}=q_{2}^{*} \tau_{t}^{11}, \\
F_{15}=q_{4}^{*}, F_{16}=\tau_{f}^{10}, F_{17}=-q_{3}^{*} \tau_{c}^{11}
\end{gathered}
$$


The system of equations (2.3) and (2.4) has a non-trivial solution if the determinant of the coefficients $\left[\bar{\psi}, \overline{\varphi_{2}}\right]^{T}$ vanishes, which yields to the following polynomial characteristic equation

$$
F_{1} \xi^{4}+F_{2} \xi^{2}+F_{3}=0
$$

where,

$$
F_{1}=-\zeta_{1}\left(1-\delta^{2}\right), F_{2}=-\zeta_{1}^{*} \zeta_{2}, F_{3}=\left(\zeta_{3}-w^{2}\right)\left(1-\delta^{2}\right)-w^{2} \zeta_{1}+w^{2}\left(\zeta_{3}-w^{2}\right)
$$

Solving (2.8) we obtain eight roots of $\xi$ in which four roots $\xi_{1}, \xi_{2}, \xi_{3}, \xi_{4}$ corresponds to positive $x_{3}$ - direction and represents the four waves in descending order of their velocities, namely LD-wave, T-wave, MD-wave, LM-wave. Likewise, solving $(2.9)$ we obtain four roots of $\xi$, in which two roots $\xi_{5}$ and $\xi_{6}$ correspond to positive $x_{3}$ - direction and other two roots $-\xi_{5}$ and $-\xi_{6}$ correspond to negative $x_{3}$ - direction. Now and after, we will restrict our work to positive $x_{3}$ - direction . Corresponding to roots $\xi_{5}$ and $\xi_{6}$ there exist two waves in descending order of their velocities, namely CD-I and CD-II waves. We now derive the expressions for phase velocity, attenuation coefficient, specific loss and penetration depth of these waves.

\section{(i) Phase velocity}

The phase velocity is given by

$$
V_{i}=\frac{\omega}{\left|R e\left(\xi_{i}\right)\right|}, \quad i=1,2,3,4,5,6
$$

where $V_{1}, V_{2}, V_{3}, V_{4}, V_{5}, V_{6}$ are the phase velocities of LD, T, MD, LM, CD-I and $\mathrm{CD}-\mathrm{II}$ waves respectively.

\section{(ii) Attenuation coefficient}

The attenuation coefficient is defined as

$$
Q_{i}=\operatorname{Im}\left(\xi_{i}\right), \quad i=1,2,3,4,5,6
$$

where $Q_{1}, Q_{2}, Q_{3}, Q_{4}, Q_{5}, Q_{6}$ are the attenuation coefficients of LD, T, MD, LM, CD-I and CD-II, waves respectively.

\section{(iii) Specific loss}

The specific loss is the ratio of energy $(\Delta W)$ dissipated in taking a specimen through a stress cycle, to the elastic energy $(W)$ stored in the specimen when the strain is a maximum. The specific loss is the most direct method of defining internal friction for a material. For a sinusoidal plane wave of small amplitude, 
Kolsky[38], shows that the specific loss $\Delta W / W$ equals $4 \pi$ times the absolute value of the imaginary part of $\xi$ to the real part of $\xi$, i.e.

$$
R_{i}=\left(\frac{\Delta W}{W}\right)_{i}=4 \pi\left|\frac{\operatorname{Im}\left(\xi_{i}\right)}{\operatorname{Re}\left(\xi_{i}\right)}\right|, \quad i=1,2,3,4,5,6
$$

where $R_{1}, R_{2}, R_{3}, R_{4}, R_{5}, R_{6}$ are the specific losses of LD, T, MD, LM, CD-I and CD-II, waves respectively.

\section{(iv) Penetration depth}

The penetration depth is defined by

$$
S_{i}=\frac{1}{\left|\operatorname{Im}\left(\xi_{i}\right)\right|}, \quad i=1,2,3,4,5,6
$$

where $S_{1}, S_{2}, S_{3}, S_{4}, S_{5}, S_{6}$ are the attenuation coefficients of LD, T, MD, LM, CD-I and CD-II, waves respectively.

\section{Particular cases}

1. In absence of microstretch effect, the equation (2.8) becomes,

$$
H_{1} \xi^{6}+H_{2} \xi^{4}+H_{3} \xi^{2}+H_{4}=0,
$$

where,

$$
\begin{gathered}
H_{1}=q_{3}^{*} \tau_{c}^{11}-q_{1}^{*} \zeta_{2}^{*} \tau_{c}^{11} \\
H_{2}=-q_{3}^{*} \tau_{t}^{10} \tau_{c}^{11}-\tau_{f}^{10}-l_{3}^{*} q_{2}^{*} \tau_{c}^{10} \tau_{t}^{11}-l_{1}^{*} q_{3}^{*} \tau_{e}^{10} \tau_{t}^{11} \tau_{c}^{11}+w^{2} q_{3}^{*} \tau_{c}^{11} \\
-l_{1}^{*} \tau_{e}^{10} \zeta_{2}^{*} \tau_{c}^{11} \tau_{t}^{11} q_{2}^{*}-l_{3}^{*} q_{1}^{*} \tau_{c}^{10} \tau_{t}^{11}+q_{1}^{*} \zeta_{2}^{*} \tau_{c}^{11}-l_{1}^{*} \tau_{e}^{10} \tau_{t}^{11} \tau_{c}^{11} q_{3}^{*}, \\
H_{3}=\tau_{t}^{10} \tau_{f}^{10}-w^{2} q_{3}^{*} \tau_{t}^{10} \tau_{c}^{11}-w^{2} \tau_{f}^{10}-w^{2} l_{3}^{*} q_{2}^{*} \tau_{c}^{10} \tau_{t}^{11}+\tau_{t}^{11} \tau_{f}^{10} l_{1}^{*} \tau_{e}^{10}, \\
H_{4}=w^{2} \tau_{f}^{10} \tau_{t}^{10}
\end{gathered}
$$

Solving (2.14) we obtain six roots of $\xi$, in which three roots $\xi_{1}, \xi_{2}$ and $\xi_{3}$ corresponds to positive $x_{3}$ - direction and represents the three waves in descending order of their velocities, namely LD-wave, T-wave, MD-wave.

2. In absence of micropolarity effect, the velocity equation (2.9) becomes

$$
\left(\delta^{2}-1\right) \xi^{2}+w^{2}=0 .
$$

Solving (2.15) we obtain two roots of $\xi$, in which one root which corresponds to positive $x_{3}-$ direction, represents the $\mathrm{SV}$-wave. 


\section{Reflection and transmission at the boundary surface}

\section{Formulation of the problem}

We consider an inviscid fluid half-space $\left(M_{1}\right)$ lying over a homogeneous isotropic, microstretch generalized thermoelastic diffusion solid half-space $\left(M_{2}\right)$. The origin of the cartesian coordinate system $\left(x_{1}, x_{2}, x_{3}\right)$ is taken at any point on the plane surface (interface) and $x_{3}$ - axis point vertically downwards into the microstretch thermoelastic diffusion solid half-space. The inviscid fluid half-space $\left(M_{1}\right)$ occupies the region $x_{3} \leq 0$ and the region $x_{3} \geq 0$ is occupied by the microstretch themoelastic diffusion solid half-space $\left(M_{2}\right)$ as shown in Figure 1 . We consider plane waves in the $x_{1}-x_{3}$ plane with wave front parallel to the $x_{2}-$ axis.

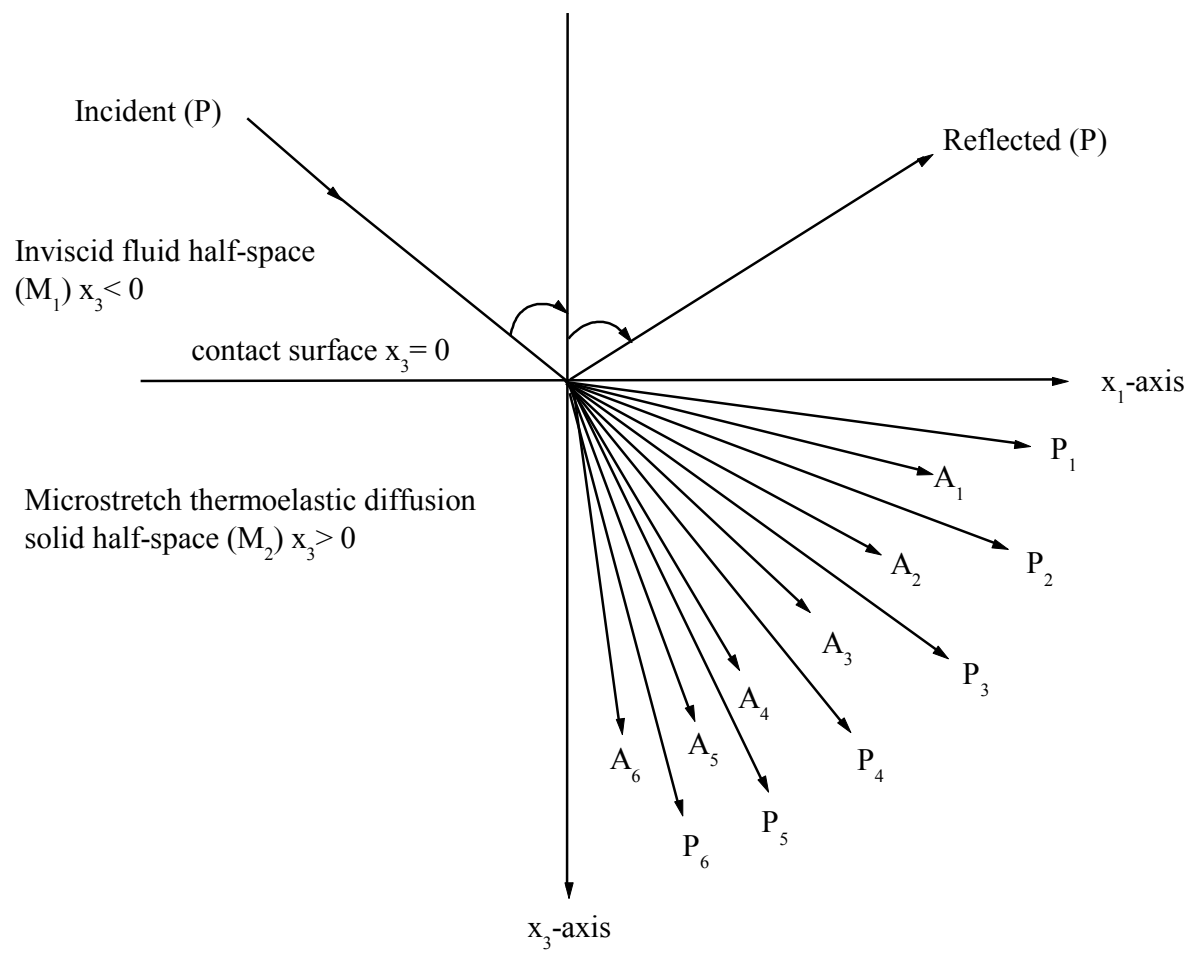

Figure 1: Geometry of the problem.

For the propagation of harmonic waves in $x_{1} x_{3}$ - plane, we assume 


$$
\left[\phi, \psi, T, C, \varphi^{*}, \varphi_{2}\right]\left(x_{1}, x_{3}, t\right)=\left[\bar{\phi}, \bar{\psi}, \bar{T}, \bar{C}, \bar{\varphi}^{*}, \bar{\varphi}_{2}\right] e^{-\iota \omega t},
$$

where $\omega$ is the angular frequency of vibrations of material particles.

Substituting the expressions of $\phi, \psi, T, C, \varphi^{*}, \varphi_{2}$ given by equation (2.1) in the equations (1.18)-(1.23), we obtain

$$
\begin{gathered}
{\left[\nabla^{2}+\omega^{2}\right] \bar{\phi}-\tau_{t}^{11} \bar{T}+\zeta_{3}^{*} \bar{\varphi}^{*}-\zeta_{2}^{*} \tau_{c}^{11} \bar{C}=0,} \\
\left(\left(1-\delta^{2}\right) \nabla^{2}+\omega^{2}\right) \bar{\psi}+\zeta_{1}^{*} \overline{\varphi_{2}}=0 \\
\zeta_{2} \nabla^{2} \bar{\psi}+\left(-\omega^{2}-\zeta_{1} \nabla^{2}+\zeta_{3}\right) \bar{\varphi}_{2}=0 \\
-\chi_{2}^{*} \nabla^{2} \bar{\phi}+\chi_{3}^{*} \tau_{t}^{11} \bar{T}+r_{1} \bar{\varphi}^{*}+r_{2} \bar{C}=0, \\
l_{1}^{*} \tau_{e}^{10} \nabla^{2} \bar{\phi}+\left(\tau_{t}^{10}-\nabla^{2}\right) \bar{T}+l_{2}^{*} \tau_{e}^{10} \bar{\varphi}^{*}+l_{3}^{*} \tau_{c}^{10} \bar{C}=0, \\
q_{1}^{*} \nabla^{4} \bar{\phi}+q_{2}^{*} \tau_{t}^{11} \nabla^{2} \bar{T}+q_{4}^{*} \nabla^{2} \bar{\varphi}^{*}+\left(\tau_{f}^{10}-q_{3}^{*} \tau_{c}^{11} \nabla^{2}\right) \bar{C}=0,
\end{gathered}
$$

where, $r_{1}=\delta_{1}^{2} \nabla^{2}-\chi_{1}^{*}+w^{2}, r_{2}=\chi_{4}^{*}(1-\iota w)$.

Eliminating $\left[\bar{\varphi}, \bar{T}, \bar{\varphi}^{*}, \bar{C}\right]^{T}$ from the system of equations (3.2), (3.5)-(3.7), we obtain

$$
\left[\nabla^{8}+B_{1} \nabla^{6}+B_{2} \nabla^{4}+B_{3} \nabla^{2}+B_{4}\right] \bar{\phi}=0,
$$

where,

$$
\begin{gathered}
B_{i}=\frac{A_{i}}{A},(i=1,2,3,4), \\
A=g_{1}^{*}-a_{14} g_{14}^{*}, A_{1}=g_{2}^{*}+g_{1}^{*} w^{2}-a_{12} g_{6}^{*}+a_{13} g_{9}^{*}-a_{14} g_{12}^{*}, \\
A_{2}=g_{3}^{*}+g_{2}^{*} w^{2}-a_{12} g_{7}^{*}+a_{13} g_{10}^{*}-a_{14} g_{13}^{*}, A_{3}=g_{4}^{*}+g_{3}^{*} w^{2}-a_{12} g_{8}^{*}+a_{13} g_{11}^{*}, A_{4}=g_{4}^{*} w^{2} \\
g_{1}^{*}=-\delta_{1}^{2} a_{46}, \\
g_{2}^{*}=a_{23} a_{46}-a_{24} a_{43}+\delta_{1}^{2}\left(a_{32} a_{46}+a_{45}+a_{34} a_{42}\right), g_{4}^{*}=a_{45}\left(a_{22} a_{33}+a_{23} a_{32}\right), \\
g_{3}^{*}=-a_{33}\left(a_{22} a_{46}+a_{24} a_{42}\right)-a_{23}\left(a_{32} a_{46}+a_{45}+a_{34} a_{42}\right) \\
+a_{43}\left(a_{24} a_{32}-a_{22} a_{34}\right)-\delta_{1}^{2} a_{32} a_{45}, g_{6}^{*}=\delta_{1}^{2}\left(a_{31} a_{46}+a_{41} a_{34}\right), \\
g_{7}^{*}=-a_{33}\left(a_{21} a_{46}+a_{24} a_{41}\right)-a_{23}\left(a_{31} a_{46}+a_{34} a_{41}\right)+a_{43}\left(a_{24} a_{31}-a_{21} a_{34}\right)-\delta_{1}^{2} a_{31} a_{45}, \\
g_{8}^{*}=a_{45}\left(a_{23} a_{31}+a_{21} a_{33}\right), g_{9}^{*}=a_{24} a_{41}+a_{21} a_{46}, \\
g_{10}^{*}=-a_{21}\left(a_{32} a_{46}+a_{45}+a_{34} a_{42}\right)+a_{22}\left(a_{31} a_{46}+a_{34} a_{41}\right)+a_{24}\left(a_{31} a_{42}-a_{32} a_{41}\right), \\
g_{11}^{*}=a_{45}\left(a_{21} a_{32}-a_{22} a_{31}\right), g_{12}^{*}=-\left(a_{23} a_{41}+a_{21} a_{43}\right)+\delta_{1}^{2}\left(a_{31} a_{42}-a_{41} a_{32}\right), \\
g_{13}^{*}=a_{33}\left(a_{22} a_{41}-a_{21} a_{42}\right)+a_{23}\left(a_{32} a_{41}-a_{31} a_{42}\right)+a_{43}\left(a_{21} a_{32}-a_{22} a_{31}\right), g_{14}^{*}=\delta_{1}^{2} a_{41}, \\
a_{11}=\nabla^{2}+w^{2}, a_{21}=-\chi_{2}^{*}, a_{31}=l_{1}^{*} \tau_{e}^{10}, a_{41}=q_{1}^{*}, a_{12}=-\tau_{t}^{11}, a_{22}=\chi_{3}^{*} \tau_{t}^{11}, \\
a_{32}=\tau_{t}^{10}, a_{42}=q_{2}^{*} \tau_{t}^{11}, a_{13}=\zeta_{3}^{*}, a_{23}=\chi_{1}^{*}-w^{2}, a_{33}=l_{2}^{*} \tau_{e}^{10},
\end{gathered}
$$




$$
\begin{gathered}
a_{14}=-\zeta_{2}^{*} \tau_{c}^{11}, a_{24}=r_{2}, a_{34}=l_{3}^{*} \tau_{c}^{10}, a_{43}=q_{4}^{*} \nabla^{2}, \\
a_{45}=\tau_{f}^{10}, a_{46}=q_{3}^{*} \tau_{c}^{11}, a_{44}=\left(a_{45}-a_{46} \nabla^{2}\right) .
\end{gathered}
$$

The general solution of equation (3.8) can be written as

$$
\bar{\phi}=\bar{\phi}_{1}+\bar{\phi}_{2}+\bar{\phi}_{3}+\bar{\phi}_{4},
$$

where the potentials $\bar{\phi}_{i}, i=1,2,3,4$ are solutions of wave equations, given by

$$
\left[\nabla^{2}+\frac{\omega^{2}}{V_{i}^{2}}\right] \bar{\phi}_{i}=0, i=1,2,3,4 .
$$

Here $V_{1}, V_{2}, V_{3}$ and $V_{4}$ are the velocities of four longitudinal waves, that is, longitudinal displacement wave (LD), thermal wave (T), mass diffusion wave (MD) and longitudinal microstretch wave (LM) and derived from the roots of the biquadratic equation in $V^{2}$, given by

$$
B_{4} V^{8}-B_{3} \omega^{2} V^{6}+B_{2} \omega^{4} V^{4}-B_{1} \omega^{6} V^{2}+w^{8}=0 .
$$

Making use of equation (3.9) in the equations (3.2), (3.5)-(3.7) with the aid of equations (3.1) and (3.10), the general solutions for $\phi, T, \varphi^{*}$, and $C$ are obtained as

$$
\left[\phi, T, \varphi^{*}, C\right]=\sum_{i=1}^{4}\left[1, k_{1 i}, k_{2 i}, k_{3 i}\right] \bar{\phi}_{i}
$$

where,

$$
\begin{gathered}
k_{1 i}=\left(g_{6}^{*} w^{6}-g_{7}^{*} w^{4} V_{i}^{2}+g_{8}^{*} w^{2} V_{i}^{4}\right) / k^{d}, k_{2 i}=-\left(g_{9}^{*} w^{6}+g_{10}^{*} w^{4} V_{i}^{2}+g_{11}^{*} w^{2} V_{i}^{4}\right) / k^{d}, \\
k_{3 i}=\left(-g_{14}^{*} w^{8}+g_{12}^{*} w^{6} V_{i}^{2}-g_{13}^{*} w^{4} V_{i}^{4}\right) /\left(V_{i}^{2} k^{d}\right), \\
k^{d}=\left(g_{1}^{*} w^{6}+g_{2}^{*} w^{4} V_{i}^{2}+g_{3}^{*} w^{2} V_{i}^{4}+g_{4}^{*} V_{i}^{6}\right), i=1,2,3,4 .
\end{gathered}
$$

Eliminating $\left[\bar{\psi}, \bar{\varphi}_{2}\right]^{T}$ from the system of equations (3.3)-(3.4), we obtain

$$
\left[\nabla^{4}+A^{*} \nabla^{2}+B^{*}\right] \bar{\psi}=0
$$

where,

$$
\begin{gathered}
A^{*}=\left(w^{2} \zeta_{1}+\zeta_{1}^{*} \zeta_{2}-\left(1-\delta^{2}\right)\left(\zeta_{3}+w^{2}\right)\right) /\left(1-\delta^{2}\right) \zeta_{1}, \\
B^{*}=w^{2}\left(w^{2}-\zeta_{3}\right) /\left(1-\delta^{2}\right) \zeta_{1},
\end{gathered}
$$

The general solution of equation (3.13) can be written as

$$
\bar{\psi}=\bar{\psi}_{5}+\bar{\psi}_{6}
$$


where the potentials $\bar{\psi}_{i}, i=5,6$ are solutions of wave equations, given by

$$
\left[\nabla^{2}+\frac{\omega^{2}}{V_{i}^{2}}\right] \bar{\psi}_{i}=0, i=5,6 .
$$

Here $\left(V_{i}, i=5,6\right)$ are the velocities of two coupled transverse displacement and microrotational (CD-I, CD-II) waves and derived from the root of quadratic equation in $V^{2}$, given by

$$
B^{*} V^{4}-A^{*} w^{2} V^{2}+w^{4}=0,
$$

Making use of equation (3.14) in the equations (3.3)-(3.4) with the aid of equations (3.1) and (3.15), the general solutions for $\psi$ and $\varphi_{2}$ are obtained as

$$
\left[\psi, \varphi_{2}\right]=\sum_{i=5}^{6}\left[1, n_{1 i}\right] \bar{\psi}_{i}
$$

where,

$$
n_{1 i}=\frac{\zeta_{2} w^{2}}{\left(\zeta_{3}-w^{2}\right) V_{i}^{2}+\zeta_{1} w^{2}}, \text { for } i=5,6
$$

Following Achenbach [39], the field equations in terms of velocity potential for inviscid fluid are

$$
\begin{gathered}
p^{f}=-\rho^{f} \dot{\phi}^{f}, \\
{\left[\nabla^{2}-\frac{1}{\alpha_{p}^{f 2}} \frac{\partial^{2}}{\partial t^{2}}\right] \phi^{f}=0,} \\
\vec{u}^{f}=\nabla \phi^{f},
\end{gathered}
$$

where $\alpha_{p}^{f 2}=\lambda^{f} / \rho^{f}$ and $\lambda^{f}$ is the bulk modulus, $\rho^{f}$ is the density of the liquid, $\vec{u}^{f}$ is the velocity vector and $p^{f}$ is the acoustic pressure of the inviscid fluid.

For two dimensional problem, $\vec{u}^{f}=\left(u_{1}^{f}, 0, u_{3}^{f}\right)$ can be written in terms of velocity potential as

$$
u_{1}^{f}=\frac{\partial \phi^{f}}{\partial x_{1}}, u_{3}^{f}=\frac{\partial \phi^{f}}{\partial x_{3}} .
$$

Applying the dimensionless quantities defined by (1.10) in equations (3.18) and (3.19) and after suppressing the primes, we obtain

$$
\begin{gathered}
p^{f}=-\zeta \dot{\phi}^{f}, \\
{\left[\nabla^{2}-\frac{1}{v_{p}^{f 2}} \frac{\partial^{2}}{\partial t^{2}}\right] \phi^{f}=0,}
\end{gathered}
$$


where,

$$
\zeta=\rho^{f} c_{1}^{2} / \beta_{1} T_{0}, v_{p}^{f}=\alpha_{p}^{f} / c_{1} .
$$

We assume the solution of (3.23) as

$$
\phi^{f}\left(x_{1}, x_{3}, t\right)=\bar{\phi}^{f} e^{-\iota w t} .
$$

Using (3.24) in (3.23), we have

$$
\left[\nabla^{2}+\frac{w^{2}}{v_{p}^{f 2}}\right] \phi^{f}=0
$$

\section{Reflection and transmission}

We consider a plane harmonic longitudinal wave $(\mathrm{P})$ propagating through the inviscid fluid half-space and is incident at the interface $x_{3}=0$ as shown in Figure 1. Corresponding to incident wave, one homogeneous longitudinal wave $(\mathrm{P})$ is reflected in inviscid fluid half-space and six inhomogeneous waves (LD, T, MD, LM, CD-I and CD-II) are transmitted in isotropic microstretch thermoelastic diffusion solid half-space.

In inviscid fluid half-space, the potential functions satisfying equation (3.25) can be written as

$$
\phi^{f}=A_{0}^{f} e^{\left[\iota \omega\left(\left(x_{1} \sin \theta_{0}+x_{3} \cos \theta_{0}\right) / v_{p}^{f}\right)-t\right]}+A_{1}^{f} e^{\left[\iota \omega\left(\left(x_{1} \sin \theta_{1}-x_{3} \cos \theta_{1}\right) / v_{p}^{f}\right)-t\right]},
$$

The coefficients $A_{0}^{f}$ and $A_{1}^{e}$ represent the amplitudes of the incident $\mathrm{P}$ and reflected $\mathrm{P}$ waves respectively.

Following Borcherdt [40], in a homogeneous isotropic microstretch thermoelastic diffusion half-space, potential functions satisfying equations (3.10) and (3.15) can be written as

$$
\begin{gathered}
{\left[\phi, T, \varphi^{*}, C\right]=\sum_{i=1}^{4}\left[1, k_{1 i}, k_{2 i}, k_{3 i}\right] B_{i} e^{\left(\vec{A}_{i} \cdot \vec{r}\right)} e^{\left\{\iota\left(\vec{P}_{i} \cdot \vec{r}-\omega t\right)\right\}},} \\
{\left[\psi, \phi_{2}\right]=\sum_{i=5}^{6}\left[1, n_{i p}\right] B_{i} e^{\left(\vec{A}_{i} \cdot \vec{r}\right)} e^{\iota\left(\vec{P}_{i} \cdot \vec{r}-\omega t\right)} .}
\end{gathered}
$$

The coefficients $B_{i}, i=1,2,3,4,5,6$ represent the amplitudes of transmitted waves. The propagation vector $\vec{P}_{i}, i=1,2,3,4,5,6$ and attenuation factor $\vec{A}_{i}, i=1,2,3,4,5,6$ are given by

$$
\vec{P}_{i}=\xi_{R} \hat{x}_{1}+d V_{i R} \hat{x}_{3}, \vec{A}_{i}=-\xi_{I} \hat{x}_{1}-d V_{i I} \hat{x}_{3}, i=1,2,3,4,5,6
$$


where,

$$
d V_{i}=d V_{i R}+\iota d V_{i I}=p \cdot v \cdot\left(\frac{\omega^{2}}{V_{i}^{2}}-\xi^{2}\right)^{1 / 2}, i=1,2,3,4,5,6 .
$$

and $\xi=\xi_{R}+\iota \xi_{I}$ is a complex wave number. The subscripts $\mathrm{R}$ and I denote the real and imaginary parts of the corresponding complex quantity and p.v. stands for the principal value of the complex quantity obtained after square root. $\xi_{R} \geq 0$ ensures propagation in the positive $x_{1}$-direction. The complex wave number $\xi$ in the microstretch thermoelastic diffusion solid medium is given by

$$
\xi=\left|\vec{P}_{i}\right| \sin \theta_{i}^{\prime}-\iota\left|\vec{A}_{i}\right| \sin \left(\theta_{i}^{\prime}-\gamma_{i}\right), i=1,2,3,4,5,6,
$$

where $\gamma_{i}, i=1,2,3,4,5,6$ is the angle between the propagation and attenuation vector and $\theta_{i}^{\prime}, i=1,2,3,4,5,6$ is the angle of refraction in medium II.

\section{Boundary conditions}

The boundary conditions are the continuity of stress and displacement components, vanishing of the gradient of temperature, mass concentration, the tangential couple stress and microstress components. Mathematically these can be written as

(i) Continuity of normal stress component

$$
t_{33}=-p^{f},
$$

(ii) Continuity of tangential stress component

$$
t_{33}=0,
$$

(iii) Continuity of normal displacement component

$$
u_{3}^{f}=u_{3},
$$

(iv)Thermally insulated boundary

$$
\frac{\partial T}{\partial x_{3}}=0
$$

(v)Impermeable boundary

$$
\frac{\partial C}{\partial x_{3}}=0
$$


(vi)Vanishing of the tangential couple stress component

$$
m_{32}=0
$$

(vii) Vanishing of the microstress component

$$
\lambda_{3}^{*}=0
$$

Making the use of potentials given by equations (3.26)-(3.28), we find that the boundary conditions are satisfied if and only if

$$
\xi_{R}=\frac{\omega \sin \theta_{0}}{\alpha}=\frac{\omega \sin \theta_{1}}{\alpha},
$$

and

$$
\xi_{I}=0,
$$

It means that waves are attenuating only in $x_{3}$-direction. From equation (3.31), it implies that if $\left|\overrightarrow{A_{i}}\right| \neq 0$, then $\gamma_{i}=\theta_{i}^{\prime}, i=1,2,3,4,5,6$, that is, attenuated vectors for the six transmitted waves are directed along the $x_{3}$-axis.

Using equations (3.26)-(3.28) in the boundary conditions (3.32)-(3.38) and with the aid of equations (1.6)-(1.10), (1.17), (3.21)-(3.22) and (3.39)-(3.40), we get a system of seven non-homogeneous equations which can be written as

$$
\sum_{j=1}^{7} d_{i j} Z_{j}=g_{i}
$$

where $Z_{j}=\left|Z_{j}\right| e^{\iota \psi_{j}^{*}},\left|Z_{j}\right|, \psi_{j}^{*}, j=1, \ldots, 6,7$ represent amplitude ratios and phase shift of reflected P-, transmitted LD-, transmitted T-, transmitted MD-, transmitted LM-, transmitted CD I -, transmitted CD II - waves to that of amplitude of incident wave, respectively.

$$
\begin{gathered}
d_{11}=\frac{-\iota \zeta_{2}^{*} \rho^{f} c_{1}^{2}}{\omega}, d_{16}=-(2 \mu+K) \frac{\xi_{R}}{\omega} \frac{d V_{5}}{\omega}, d_{17}=-(2 \mu+K) \frac{\xi_{R}}{\omega} \frac{d V_{6}}{\omega}, \\
d_{21}=0, d_{26}=\mu\left[\left(\frac{\xi_{R}}{\omega}\right)^{2}-\left(\frac{d V_{5}}{\omega}\right)^{2}\right]+K\left(\frac{d V_{5}}{\omega}\right)^{2}-\frac{K n_{5}^{p}}{\omega^{2}}, \\
d_{27}=\mu\left[\left(\frac{\xi_{R}}{\omega}\right)^{2}-\left(\frac{d V_{6}}{\omega}\right)^{2}\right]+K\left(\frac{d V_{6}}{\omega}\right)^{2}-\frac{K n_{6}^{p}}{\omega^{2}}, d_{31}=\frac{\iota d V_{\alpha}}{\omega}, \\
d_{36}=0, d_{37}=0, d_{41}=0, d_{46}=0, d_{47}=0, d_{51}=0, d_{56}=0, d_{57}=0, d_{61}=0, d_{71}=0, \\
d_{1 j}=-\lambda\left(\frac{\xi_{R}}{\omega}\right)^{2}-\rho c_{1}^{2}\left(\frac{d V_{j}}{\omega}\right)^{2}-\rho c_{1}^{2}\left(\frac{k_{1 j} \tau_{t}^{11}+\zeta_{2}^{*} k_{3 j} \tau_{c}^{11}}{\omega^{2}}\right)+\lambda_{0} \frac{k_{2 j}}{\omega^{2}}, \\
d_{2 j}=(2 \mu+K)\left(\frac{\xi_{R}}{\omega}\right)\left(\frac{d V_{j}}{\omega}\right), d_{3 j}=-\left(\frac{d V_{j-1}}{\omega}\right),
\end{gathered}
$$




$$
\begin{gathered}
d_{4 j}=k_{1(j-1)}\left(\frac{d V_{j-1}}{\omega}\right), d_{5 j}=k_{3(j-1)}\left(\frac{d V_{j-1}}{\omega}\right), d_{6 j}=b_{0} k_{2(j-1)} \frac{\xi_{R}}{\omega}, \\
d_{7 j}=\alpha_{0} k_{2(j-1)} \frac{d V_{j-1}}{\omega}, \text { for } j=2,3,4,5, \\
d_{6 j}=\gamma \frac{d V_{j-2}}{\omega} n_{(j-1) p}, d_{7 j}=-b_{0} n_{(j-1) p} \frac{\xi_{R}}{\omega}, \text { for } j=6,7, \\
\frac{d V_{\alpha}}{\omega}=\left(\frac{1}{\alpha^{2}}-\left(\frac{\xi_{R}}{\omega}\right)^{2}\right)^{1 / 2}=\left(\frac{1}{\alpha^{2}}-\left(\frac{\sin \theta_{0}}{\alpha}\right)^{2}\right)^{1 / 2},
\end{gathered}
$$

and

$$
\frac{d V_{j}}{\omega}=p \cdot v \cdot\left(\frac{1}{V_{j}^{2}}-\left(\frac{\sin \theta_{0}}{\alpha}\right)^{2}\right)^{1 / 2}, j=1,2,3,4,5,6,7 .
$$

Here p.v. is evaluated with restriction $d V_{j I} \geq 0$ to satisfy decay condition in the microstretch thermoelastic diffusion medium. The coefficients $g_{i}, i=1, \ldots, 7$ on right side of the equation (3.41) are given by

$$
g_{i}=(-1)^{i} d_{1 i}, \text { for } i=1,3 \text { and } g_{i}=0, \text { for } i=2,4,5,6,7
$$

Now we consider a surface element of unit area at the interface between two media. The reason for this consideration is to calculate the partition of energy of the incident wave among the reflected and transmitted waves on the both sides of surface. Following Achenbach [39], the energy flux across the surface element, that is, rate at which the energy is communicated per unit area of the surface is represented as

$$
P^{*}=t_{l m} l_{m} \dot{u}_{l}
$$

where $t_{l m}$ is the stress tensor, $l_{m}$ are the direction cosines of the unit normal $\hat{\boldsymbol{l}}$ outward to the surface element and $\dot{u}_{l}$ are the components of the particle velocity.

The time average of $P^{*}$ over a period, denoted by $\left\langle P^{*}>\right.$, represents the average energy transmission per unit surface area per unit time. Thus, on the surface with normal along $x_{3}$-direction, the average energy intensities of the waves in the inviscid fluid half-space are given by

$$
<P^{* f}>=\operatorname{Re}<p>^{f} \cdot \operatorname{Re}\left(u_{3}^{f}\right) .
$$

Following Achenbach [39], for any two complex functions $f$ and $g$, we have

$$
<\operatorname{Re}(f) \cdot \operatorname{Re}(g)>=\frac{1}{2} \operatorname{Re}(f \cdot \bar{g}) .
$$


The expressions for energy ratios $E_{1}$ for the reflected P- is given by

$$
E_{1}=-\frac{<P^{* f}>}{<P_{0}^{* f}>}
$$

where

$$
<P_{1}^{* f}>=\frac{\omega^{2} \zeta_{2}^{*} \rho^{f} c_{1}^{2}}{2 \beta_{1} T_{0} v_{p}^{f}}\left|Z_{1}\right|^{2} \operatorname{Re}\left(\cos \theta_{1}\right),
$$

and

For incident $\mathrm{P}$-wave

$$
<P_{0}^{* f}>=\frac{\omega^{2} \zeta_{2}^{*} \rho^{f} c_{1}^{2}}{2 \beta_{1} T_{0} v_{p}^{f}} \cos \theta_{0},
$$

are the average energy intensities of the reflected $\mathrm{P}$ - and incident $\mathrm{P}$-waves respectively. In equation (3.46), negative sign is taken because the direction of reflected waves is opposite to that of incident wave.

For microstretch thermoelastic diffusion solid half-space, the average energy intensities of the waves on the surface with normal along $x_{3}$-direction, are given by

$$
\begin{gathered}
<P_{i j}^{*}>=\operatorname{Re}<t>_{13}^{(i)} \cdot \operatorname{Re}\left(\dot{u}_{1}^{(j)}\right)+\operatorname{Re}<t>_{33}^{(i)} \cdot \operatorname{Re}\left(\dot{u}_{3}^{(j)}\right) \\
+\operatorname{Re}<m>_{32}^{(i)} \cdot \operatorname{Re}\left(\dot{\phi}_{2}^{(j)}\right)+\operatorname{Re}<\lambda_{3}^{*}>^{(i)} \cdot \operatorname{Re}\left(\dot{\varphi}^{*(j)}\right) .
\end{gathered}
$$

The expressions for energy ratios $E_{i j}, i, j=1,2,3,4,5,6$ for the transmitted waves are given by

$$
E_{i j}=\frac{\left.<P_{i j}^{*}\right\rangle}{\left.<P_{0}^{* f}\right\rangle}, i, j=1,2,3,4,5,6,
$$

where,

$$
\begin{gathered}
<P_{i j}^{*}>=-\frac{\omega^{4}}{2} \operatorname{Re}\left[(2 \mu+K) \frac{d V_{i}}{\omega} \frac{\xi_{R}}{\omega} \frac{\bar{\xi}_{R}}{\omega}+\left\{\lambda\left(\frac{\xi_{R}}{\omega}\right)^{2}+\rho c_{1}^{2}\left(\frac{d V_{i}}{\omega}\right)^{2}\right.\right. \\
\left.\left.+\frac{\rho c_{1}^{2}\left(k_{1 i} \tau_{t}^{11}+\zeta_{2}^{*} k_{3 i} \tau_{c}^{11}\right)}{\omega^{2}}-\frac{\lambda_{0} k_{2 i}}{\omega^{2}}\right\} \frac{d V_{j}}{\omega}+\frac{\alpha_{0} k_{2 i} \bar{k}_{2 j} w^{* 2}}{\rho \omega^{2} c_{1}^{4}} \frac{d V_{i}}{\omega}\right] Z_{i+2} \bar{Z}_{j+2}, \\
i, j=1,2,3,4 . \\
<P_{i j}^{*}>=-\frac{\omega^{4}}{2} \operatorname{Re}\left[(2 \mu+K) \frac{d V_{i}}{\omega} \frac{\xi_{R}}{\omega} \frac{\bar{\xi}_{R}}{\omega}-\left\{\mu\left(\frac{\xi_{R}}{\omega}\right)^{2}-(\mu+K)\left(\frac{d V_{i}}{\omega}\right)^{2}+\frac{K n_{i p}}{\omega^{2}}\right\} \frac{d V_{j}}{\omega}+\right.
\end{gathered}
$$




$$
\begin{aligned}
& \left.\frac{\gamma n_{i p} \bar{n}_{j p} w^{* 2}}{\rho \omega^{2} c_{1}^{4}} \frac{d V_{i}}{\omega}\right] Z_{i+2} \bar{Z}_{j+2},, i, j=5,6 . \\
& <P_{i 5}^{*}>=-\frac{\omega^{4}}{2} \operatorname{Re}\left[-(2 \mu+K) \frac{d V_{i}}{\omega} \frac{\xi_{R}}{\omega} \frac{d V_{5}}{\omega}+\left\{\lambda\left(\frac{\xi_{R}}{\omega}\right)^{2}+\rho c_{1}^{2}\left(\frac{d V_{i}}{\omega}\right)^{2}\right.\right. \\
& \left.\left.+\frac{\rho c_{1}^{2}\left(k_{1 i} \tau_{t}^{11}+\zeta_{2}^{*} k_{3 i} \tau_{c}^{11}\right)}{\omega^{2}}-\frac{\lambda_{0} k_{2 i}}{\omega^{2}}\right\} \frac{\bar{\xi}_{R}}{\omega}-\frac{b_{0} k_{2 i} \bar{n}_{5 p} w^{* 2}}{\rho \omega^{2} c_{1}^{4}} \frac{\xi_{R}}{\omega}\right] Z_{i+2} \bar{Z}_{7},, i, j=1,2,3,4 . \\
& <P_{i 5}^{*}>=-\frac{\omega^{4}}{2} \operatorname{Re}\left[(2 \mu+K) \frac{d V_{i}}{\omega} \frac{\xi_{R}}{\omega} \frac{\bar{\xi}_{R}}{\omega}-\left\{\mu\left(\frac{\xi_{R}}{\omega}\right)^{2}-(\mu+K)\left(\frac{d V_{i}}{\omega}\right)^{2}+\frac{K n_{i p}}{\omega^{2}}\right\} \frac{d V_{5}}{\omega}-\right. \\
& \left.\frac{\gamma n_{i p} \bar{n}_{5 p} w^{* 2}}{\rho \omega^{2} c_{1}^{4}} \frac{d V_{i}}{\omega}\right] Z_{i+2} \bar{Z}_{7},, i, j=5,6 \\
& <P_{i 6}^{*}>=-\frac{\omega^{4}}{2} \operatorname{Re}\left[-(2 \mu+K) \frac{d V_{i}}{\omega} \frac{\xi_{R}}{\omega} \frac{d V_{6}}{\omega}+\left\{\lambda\left(\frac{\xi_{R}}{\omega}\right)^{2}+\rho c_{1}^{2}\left(\frac{d V_{i}}{\omega}\right)^{2}\right.\right. \\
& \left.\left.+\frac{\rho c_{1}^{2}\left(k_{1 i} \tau_{t}^{11}+\zeta_{2}^{*} k_{3 i} \tau_{c}^{11}\right)}{\omega^{2}}-\frac{\lambda_{0} k_{2 i}}{\omega^{2}}\right\} \frac{\bar{\xi}_{R}}{\omega}-\frac{b_{0} k_{2 i} \bar{n}_{6 p} w^{* 2}}{\rho \omega^{2} c_{1}^{4}} \frac{\xi_{R}}{\omega}\right] Z_{i+2} \bar{Z}_{8},, i, j=1,2,3,4 . \\
& <P_{i 6}^{*}>=-\frac{\omega^{4}}{2} \operatorname{Re}\left[(2 \mu+K) \frac{d V_{i}}{\omega} \frac{\xi_{R}}{\omega} \frac{\bar{\xi}_{R}}{\omega}-\left\{\mu\left(\frac{\xi_{R}}{\omega}\right)^{2}-(\mu+K)\left(\frac{d V_{i}}{\omega}\right)^{2}+\frac{K n_{i p}}{\omega^{2}}\right\} \frac{d V_{6}}{\omega}-\right. \\
& \left.\frac{\gamma n_{i p} \bar{n}_{6 p} w^{* 2}}{\rho \omega^{2} c_{1}^{4}} \frac{d V_{i}}{\omega}\right] Z_{i+2} \bar{Z}_{8},, i, j=5,6 \\
& <P_{5 j}^{*}>=-\frac{\omega^{4}}{2} \operatorname{Re}\left[(2 \mu+K) \frac{d V_{5}}{\omega} \frac{\xi_{R}}{\omega} \frac{d V_{j}}{\omega}+\left\{\mu\left(\frac{\xi_{R}}{\omega}\right)^{2}-(\mu+K)\left(\frac{d V_{5}}{\omega}\right)^{2}+\frac{K n_{5 p}}{\omega^{2}}\right\} \frac{\bar{\xi}_{R}}{\omega}-\right. \\
& \left.\frac{b_{0} n_{5 p} \bar{k}_{2 j} w^{* 2}}{\rho \omega^{2} c_{1}^{4}} \frac{\xi_{R}}{\omega}\right] Z_{7} \bar{Z}_{j+2},, i, j=1,2,3,4 \text {. } \\
& <P_{5 j}^{*}>=-\frac{\omega^{4}}{2} \operatorname{Re}\left[(2 \mu+K) \frac{d V_{5}}{\omega} \frac{\xi_{R}}{\omega} \frac{\bar{\xi}_{R}}{\omega}-\left\{\mu\left(\frac{\xi_{R}}{\omega}\right)^{2}-(\mu+K)\left(\frac{d V_{j}}{\omega}\right)^{2}+\frac{K n_{5 p}}{\omega^{2}}\right\} \frac{d V_{j}}{\omega}+\right. \\
& \left.\frac{\gamma n_{5 p} \bar{n}_{j p} w^{* 2}}{\rho \omega^{2} c_{1}^{4}} \frac{d V_{5}}{\omega}\right] Z_{7} \bar{Z}_{j+2},, i, j=5,6 \\
& <P_{6 j}^{*}>=-\frac{\omega^{4}}{2} \operatorname{Re}\left[(2 \mu+K) \frac{d V_{6}}{\omega} \frac{\xi_{R}}{\omega} \frac{d V_{j}}{\omega}+\left\{\mu\left(\frac{\xi_{R}}{\omega}\right)^{2}-(\mu+K)\left(\frac{d V_{6}}{\omega}\right)^{2}+\frac{K n_{6 p}}{\omega^{2}}\right\} \frac{\bar{\xi}_{R}}{\omega}-\right.
\end{aligned}
$$




$$
\begin{gathered}
\left.\frac{b_{0} n_{6 p} \bar{k}_{2 j} w^{* 2}}{\rho \omega^{2} c_{1}^{4}} \frac{\xi_{R}}{\omega}\right] Z_{8} \bar{Z}_{j+2},, i, j=1,2,3,4 . \\
<P_{6 j}^{*}>=-\frac{\omega^{4}}{2} \operatorname{Re}\left[(2 \mu+K) \frac{d V_{6}}{\omega} \frac{\xi_{R}}{\omega} \frac{\bar{\xi}_{R}}{\omega}-\left\{\mu\left(\frac{\xi_{R}}{\omega}\right)^{2}-(\mu+K)\left(\frac{d V_{j}}{\omega}\right)^{2}+K n_{j p}\right\} \frac{d \bar{V}_{j}}{\omega}+\right. \\
\left.\frac{\gamma n_{6 p} \bar{n}_{j p} w^{* 2}}{\rho \omega^{2} c_{1}^{4}} \frac{d V_{6}}{\omega}\right] Z_{8} \bar{Z}_{j+2},, i, j=5,6 .
\end{gathered}
$$

The diagonal entries of energy matrix $E_{i j}$ in equation (3.49) represent the energy ratios of the waves, whereas sum of the non-diagonal entries of $E_{i j}$ give the share of interaction energy among all the transmitted waves in the medium and is given by

$$
E_{R R}=\sum_{i=1}^{6}\left(\sum_{j=1}^{6} E_{i j}-E_{i i}\right) .
$$

The energy ratios $E_{1}$, diagonal entries and sum of non-diagonal entries of energy matrix $E_{i j}$, that is, $E_{11}, E_{22}, E_{33}, E_{44}, E_{55}, E_{66}$ and $E_{R R}$ yield the conservation of incident energy across the interface, through the relation

$$
E_{1}+E_{11}+E_{22}+E_{33}+E_{44}+E_{55}+E_{66}+E_{R R}=1 .
$$

\section{Special Case}

In absence of microstretch effect in equations (3.41), (3.46) and (3.49), we obtain the corresponding amplitude and energy ratios at the interface of inviscid fluid half-space and micropolar thermoelastic diffusion solid half-space.

\section{Numerical results and Discussion}

The analysis is conducted for a magnesium crystal-like material.Following [41], the values of physical constants are

$$
\begin{gathered}
\lambda=9.4 \times 10^{10} \mathrm{Nm}^{-2}, \mu=4.0 \times 10^{10} \mathrm{Nm}^{-2}, K=1.0 \times 10^{10} \mathrm{Nm}^{-2}, \\
\rho=1.74 \times 10^{3} \mathrm{Kgm}^{-3}, \gamma=0.779 \times 10^{-9} \mathrm{~N}, j=0.2 \times 10^{-19} \mathrm{~m}^{2},
\end{gathered}
$$

Thermal and diffusion parameters are given by

$$
\begin{gathered}
C^{*}=1.04 \times 10^{3} \mathrm{JKg}^{-1} \mathrm{~K}^{-1}, K^{*}=1.7 \times 10^{6} \mathrm{Jm}^{-1} \mathrm{~s}^{-1} \mathrm{~K}^{-1}, \alpha_{t 1}=2.33 \times 10^{-5} \mathrm{~K}^{-1}, \\
\alpha_{t 2}=2.48 \times 10^{-5} \mathrm{~K}^{-1}, \alpha_{c 1}=2.65 \times 10^{-4} \mathrm{~m}^{3} \mathrm{Kg}^{-1}, \alpha_{c 2}=2.83 \times 10^{-4} \mathrm{~m}^{3} \mathrm{Kg}^{-1}, \\
T_{0}=0.298 \times 10^{3} \mathrm{~K}, a=2.9 \times 10^{4} \mathrm{~m}^{2} \mathrm{~s}^{-2} \mathrm{~K}^{-1}, b=32 \times 10^{5} \mathrm{Kg}^{-1} \mathrm{~m}^{5} \mathrm{~s}^{-2},
\end{gathered}
$$




$$
D=0.85 \times 10^{-8} \mathrm{Kgm}^{-3} \mathrm{~s},
$$

The values of relaxation times are taken as:

$$
\tau_{0}=0.02 s, \tau_{1}=0.01 s, \tau^{0}=0.03 s, \tau^{1}=0.04 s .
$$

and the microstretch parameters are taken as

$$
\begin{gathered}
j_{0}=0.19 \times 10^{-19} \mathrm{~m}^{2}, \alpha_{0}=0.779 \times 10^{-9} \mathrm{~N}, b_{0}=0.5 \times 10^{-9} \mathrm{~N}, \\
\lambda_{0}=0.5 \times 10^{10} \mathrm{Nm}^{-2}, \lambda_{1}=0.5 \times 10^{10} \mathrm{Nm}^{-2},
\end{gathered}
$$

The fluid parameters are taken as:

$$
\lambda^{f}=2.1904 \times 10^{9} \mathrm{Kgm}^{-1} \mathrm{~s}^{-2}, \rho^{f}=1.0 \times 10^{3} \mathrm{Kgm}^{-1} \mathrm{~s}^{-2},
$$

The software Matlab 7.0.4 has been used to determine the values of phase velocity, attenuation coefficient, specific loss and penetration depth of plane waves, i.e. LD, T, MD, LM, CD-I and CD-II. The variations of phase velocity, attenuation coefficients, specific loss and penetration depth with respect to frequency has been shown in Figures1.1-1.6, 1.7-1.10,1.11-1.14 and 1.15-1.18 respectively. In all the Figures, MSLS and MSGL corresponds respectively to L-S and G-L theory of microstretch generalized thermoelastic diffusion solid whereas MLLS and MLGL corresponds respectively to L-S and G-L theory of micropolar generalized thermoelastic diffusion solid. In the Figures 1.1-1.4 and Figs 1.7-1.18, slant line, horizontal square, horizontal line and slant square corresponds to MSLS, MLLS, MSGL and MLGL. In Figure 1.6, vertical and slant lines correspond to phase velocities of CDI and SV waves respectively whereas vertical line in Figure 1.7 is for the phase velocity of CDII wave.

The software Matlab 7.0.4 has been used to determine the values of phase velocity, attenuation coefficient, specific loss and penetration depth of plane waves, i.e. LD, T, MD, LM, CD-I and CD-II. The variations of phase velocity, attenuation coefficients, specific loss and penetration depth with respect to frequency has been shown in Figures 2-7,8-11,12-15 and 16-19 respectively. In all the Figures, MSLS and MSGL corresponds respectively to L-S and G-L theory of microstretch generalized thermoelastic diffusion solid whereas MLLS and MLGL corresponds respectively to L-S and G-L theory of micropolar generalized thermoelastic diffusion solid. In the Figures 2-5 and Figures 8-19, slant line, horizontal square, horizontal line and slant square corresponds to MSLS, MLLS, MSGL and MLGL. In Figure 6, vertical and slant lines correspond to phase velocities of CDI and SV waves respectively whereas vertical line in Figure 7 is for the phase velocity of CDII wave.

Also using software Matlab 7.0.4, the values of energy ratio $E_{1}$ and energy matrix $E_{i j}, 1, j=1,2,3,4,5,6$ defined in the previous section for different values of incident angle $\left(\theta_{0}\right)$ ranging from 0 to $90^{\circ}$ are determined for fixed frequency $\omega=2 \times \pi \times 100 H z$. Corresponding to incident $\mathrm{P}$ wave, the variation 
of energy ratios with respect to angle of incident have been plotted in Figures 20-27. In these Figures of microstretch thermoelastic diffusion medium the graphs for L-S and G-L theories are represented by the word MSLS and MSGL respectively.

\section{Phase Velocity}

Figures 2-7 show the variation of phase velocities $\left(V_{1}, V_{2}, V_{3}, V_{4}, V_{5}, V_{6}\right)$ of different waves with respect to $\omega$. It is clear from Figure 2 that phase velocity $V_{1}$ increases continuously for both LS and GL theories under the effect of microstretch. In absence of microstretch effect $V_{1}$ increase but with small difference in magnitudde values. Also the values obtained by LS theory are higher in comparison to GL theory. Figure 3 depicts that phase velocity $V_{2}$ initially increase but then shows the constant behavior for both LS and GL theories under the effect of microstretch whereas in absence of microstretch, it first increase sharply and then decreases. The values of phase velocity for LS theory remains more in comparison to the GL theory. Figure 4 exhibits that initially for LS theory, phase velocity $V_{3}$ decrease sharply but increase smoothly as $\omega$ increases further whereas in absence of microstretch effect, it increases continuously. Figure 5 depicts the variation of phase velocity $V_{4}$ for micropolar thermoelastic diffusion. It shows that $V_{4}$ initially fluctuates but increase for large values of $\omega$. Also it attains higher values for LS theory in comparison to GL theory. It is clear from Figure 6 phase velocity $V_{5}$ shows small variation for CDI and SV waves and values of $V_{5}$ for CDI wave remain more in comparison to SV wave. Figure 7 shows that for CDII wave, phase velocity $V_{6}$ increase continuously with increase in $\omega$. The values of phase velocity $V_{1}$ for MSLS and MSGL are demagnified by dividing the original values by 10 and the values of phase velocity $V_{2}$ for MLLS and MLGL are defagnified on dividing by 100 . Also the values of $V_{6}$ are magnified by multiplying with $10^{5}$.

\section{Attenuation Coefficient}

Figures 8-11 show the variation of attenuation coefficient of different waves with respect to $\omega$. Figure 8 shows that attenuation coefficient $Q_{1}$ increase sharply for microstretch thermoelastic diffusion whereas in absence of microstretch, it increase initially and then becomes constant. Figure 9 shows that the attenuation coefficient $Q_{2}$ increase sharply in absence of microstretch effect whereas for microstretch thermoelastic diffusion, it increase initially and then becomes constant. It is clear from Figure 10 that for microstretch thermoelastic diffusion attenuation coefficient $Q_{3}$ initially decrease but increase continuously for large values of $\omega$. But in absence of microstretch effect, $Q_{3}$ shows the negligible variation. Figure 11 depicts that attenuation coefficient 
$Q_{4}$ increase sharply for small values of $\omega$ and shows constant behavior for large values of $\omega$. The values of attenuation coefficient $Q_{1}$ for MSLS and MSGL are contracted by dividing with 10 .

\section{Specific Loss}

Figures 12-15 show the variation of specific loss of different waves with respect to $\omega$. Figure 12 shows that specific loss $R_{1}$ decrease sharply for small values of $\omega$ but decrease with small variation as increases further for LS and GL theories of microstretch thermoelastic diffusion medium. In absence of microstretch effect $R_{1}$ increases for LS theory and decreases for GL theory. Figure 13 depicts that values of $R_{2}$ increase initially and then shows minimum variation for microstretch thermoelastic diffusion medium whereas for micropolar thermoelastic diffusion medium, it increases continuously. The values of $R_{2}$ for LS theory remains more in comparison to GL theory. Figure 14 shows that values of $R_{3}$ first decrease sharply and then shows minimum variation and appears to be constant. The values of $R_{3}$ for microstretch theory remains less in comparison to micropolar thermoelastic diffusion theory. It is clear from Figure 15 that for microstretch thermoelastic diffusion medium with GL theory, the values of $R_{4}$ increase continuously whereas for LS theory, $R_{4}$ shows a small increase initially and constant behavior is observed for large values of $\omega$. The values of specific loss $R_{1}$ for MSLS and MSGL are demagnified on dividing by $10^{3}$. Also the values of $R_{2}$ for MLLS and MLGL are divided by $10^{3}$.

\section{Penetration Depth}

Figures 16-19 show the variation of penetration depth of different waves with respect to $\omega$. Figure 16 shows that for microstretch thermoelastic diffusion theory, the values of $S_{1}$ decrease smoothly and shows constant behavior for large values of $\omega$. In absence of microstretch effect, minimum variation is observed. It is clear from Figure 17 that for small values of $\omega$, values of $S_{2}$ decrease sharply and becomes constant as $\omega$ increases further. The variation in values of $S_{2}$ remains similar for MSLS, MLLS, MSGL and MLGL theories with difference in their magnitude values. Figure 18 shows that the behavior and variation in values of $S_{3}$ is similar as shown by $S_{2}$ in Figure15 but with different magnitude values. Figure 19 exhibits that for small values of $\omega, S_{4}$ decrease sharply for microstretch thermoelastic diffusion medium and becomes constant as $\omega$ increases further.

\section{Energy Ratios}

Figures 20-27 depicts the variation of energy ratios with the angle of incidence $\theta_{0}$. 
Figure 20 exhibits the variation of energy ratio $E_{1}$ with the angle of incidence $\theta_{0}$. It shows that the values of $E_{1}$ for both cases MDLS and MDGL decrease with the increase in $\theta_{0}$ from 0 to $20^{\circ}$ and then increase sharply for $20^{0}<\theta_{0} \leq 30^{0}$ and further decrease slowly and appears to be constant. The maximum and minimum value of $E_{1}$ for MDLS are higher than MDGL.

Figure 21 depicts the variation of energy ratio $E_{11}$ with $\theta_{0}$ and it shows that the values of $E_{11}$ for the case of MDGL are similar to MDLS but the corresponding values are different in magnitude. It is observed that there is a sharp increase and decrease in the values for 0 to $20^{\circ}$ and then decrease with small variation in the magnitude values. Values for MDGL are higher than MDLS.

Figure 22 exhibits the variation of energy ratio $E_{22}$ with $\theta_{0}$ and it shows that the maximum value of $E_{22}$ is attained for MDGL. Also it increase sharply at $\theta_{0}=6.5^{0}$ after that shows sudden decrease and variation in values is small for $\theta_{0}>20^{0}$.

Figure 23 depicts the variation of energy ratio $E_{33}$ with $\theta_{0}$ and it indicates the values of $E_{33}$ for the case of MDLS are large as compared to the MDGL within the whole range of $\theta_{0}$, though the maximum value of $E_{33}$ can be noticed within the range $0 \leq \theta_{0} \leq 30^{\circ}$ for both the cases.

Figure 24 depicts the variation of energy ratio $E_{44}$ with $\theta_{0}$. It shows that the values of $E_{44}$ for both cases MDLS and MDGL increase initially with the increase of $\theta_{0}$ from 0 to $7^{0}$ and then decrease as $\theta_{0}$ increase further. The value of $E_{44}$ in case of MDGL are higher than MDLS.

Figure 25 exhibits the variation of energy ratio $E_{55}$ with $\theta_{0}$ and it indicates the behavior of the graph is nearly equivalent to that of Figure 24 but the corresponding values are different in magnitude.

Figure 26 shows the variations of $E_{66}$ with $\theta_{0}$ and it indicates that the value of $E_{66}$ for both MDLS and MDGL shows the same behavior and variation as $E_{33}$ but with different magnitude values.

Figure 27 shows the variation of interaction energy ratio $E_{R R}$ with $\theta_{0}$ and it indicates the values of $E_{R R}$ for the case of MDLS are less as compared to MDGL within the whole range of $\theta_{0}$. The values of interaction energy decrease initially for $0 \leq \theta_{0} \leq 5^{0}$ thereafter increase sharply at $\theta_{0}=7^{0}$ and for $\theta_{0}>7^{0}$, increase in values is minimum and attain value nearly to zero.

\section{Conclusions}

The propagation of plane waves in a homogeneous, isotropic microstretch generalized thermoelastic diffusion solid medium of infinite extent is studied. The phenomenon of reflection and refraction of obliquely incident elastic waves at the interface between an inviscid fluid half-space and a microstretch thermoelastic diffusion solid half-space has also been studied. The six waves 
in microstretch thermoelastic diffusion medium are identified and explained through different wave equations in terms of displacement potentials.

The phase velocities, attenuation coefficients, specific loss and penetration depth of longitudinal waves are computed and presented graphically with respect to frequency. The values of $V_{1}, Q_{1}, R_{1}$ and $S_{1}$ remains more for microstretch thermoelastic diffusion medium whereas for other roots values remains more in absence of microstretch effect.

The energy ratios of different reflected and transmitted waves to that of incident wave are computed numerically and presented graphically with respect to the angle of incidence.

From numerical results, we conclude that the effect of angle of incidence on the energy ratios of the reflected and transmitted waves is significant. It is evident that, the values of energy ratios attained their optimum values within the range $0 \leq \theta_{0}<20^{0}$ in almost all Figs related to L-S and G-L theories. In the range $20^{0} \leq \theta_{0}<90^{0}$ variation in values of energy ratios is less as compared to $0 \leq \theta_{0}<20^{0}$, where the rapid variation is observed. Moreover, the magnitude of energy ratios , $E_{1}, E_{33}, E_{66}$, for L-S theory are more as compared to G-L theory and for the other energy ratios, behavior of L-S and G-L theories is opposite. The sum of all energy ratios of the reflected waves, transmitted waves and interference between transmitted waves is verified to be always unity which ensures the law of conservation of incident energy at the interface. 


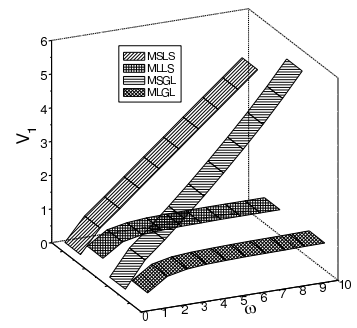

Figure 2: Variations of phase velocity $V_{1}$ with respect to frequency $\omega$

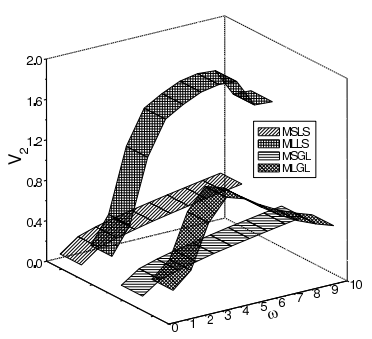

Figure 3: Variations of phase velocity $V_{2}$ with respect to frequency $\omega$ 


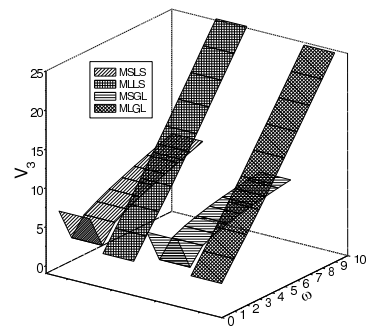

Figure 4: Variations of phase velocity $V_{3}$ with respect to frequency $\omega$

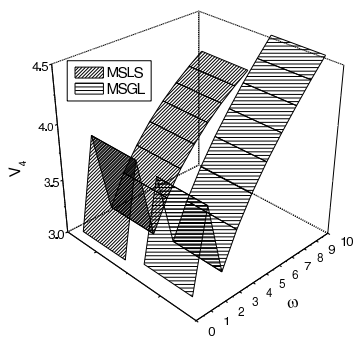

Figure 5: Variations of phase velocity $V_{4}$ with respect to frequency $\omega$ 


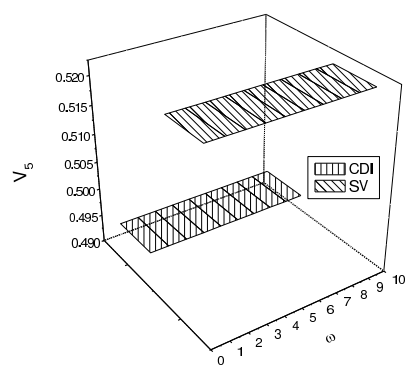

Figure 6: Variations of phase velocity $V_{5}$ with respect to frequency $\omega$

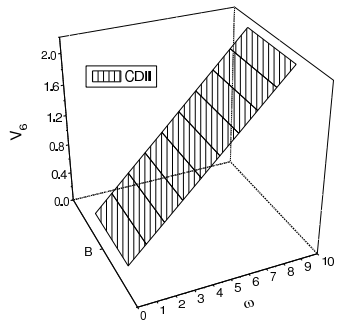

Figure 7: Variations of phase velocity $V_{6}$ with respect to frequency $\omega$ 
WAVE PROPAGATION IN A MICROSTRETCH THERMOELASTIC

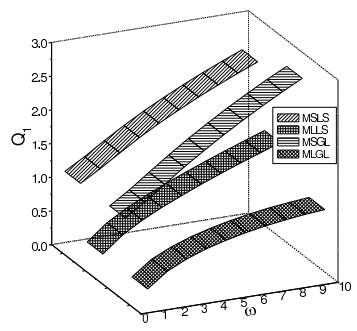

Figure 8: Variations of phase velocity $Q_{1}$ with respect to frequency $\omega$

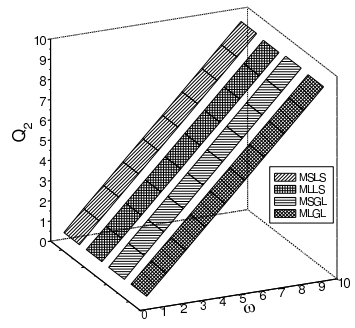

Figure 9: Variations of phase velocity $Q_{2}$ with respect to frequency $\omega$ 


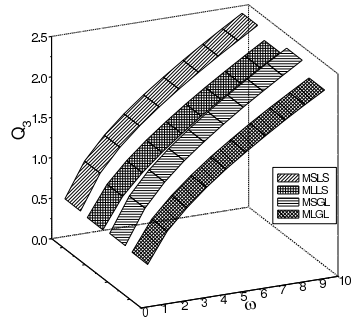

Figure 10: Variations of phase velocity $Q_{3}$ with respect to frequency $\omega$

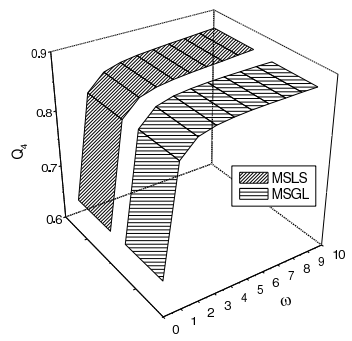

Figure 11: Variations of phase velocity $Q_{4}$ with respect to frequency $\omega$ 


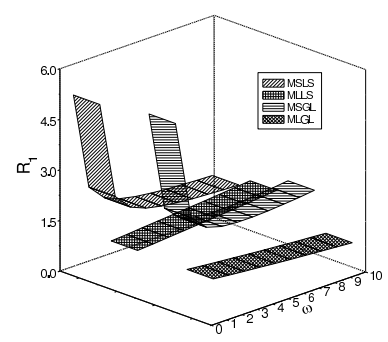

Figure 12: Variations of phase velocity $R_{1}$ with respect to frequency $\omega$

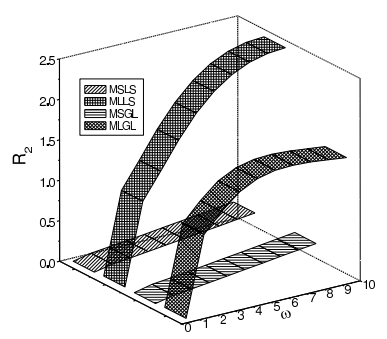

Figure 13: Variations of phase velocity $R_{2}$ with respect to frequency $\omega$ 


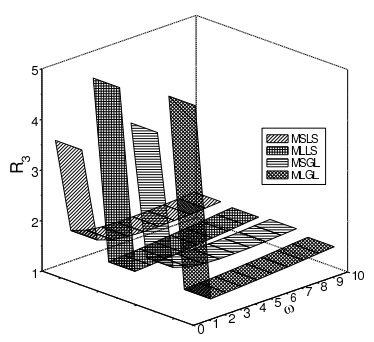

Figure 14: Variations of phase velocity $R_{3}$ with respect to frequency $\omega$

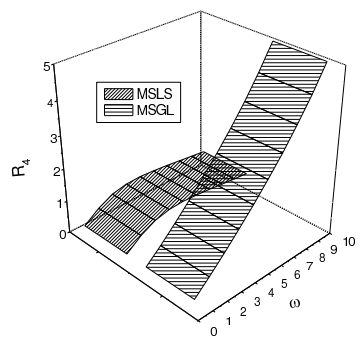

Figure 15: Variations of phase velocity $R_{4}$ with respect to frequency $\omega$ 


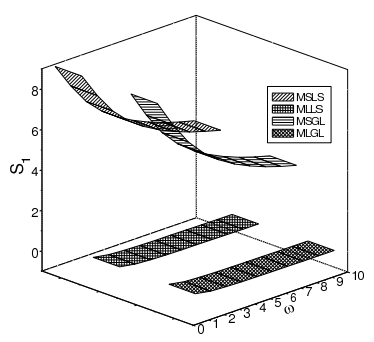

Figure 16: Variations of phase velocity $S_{1}$ with respect to frequency $\omega$

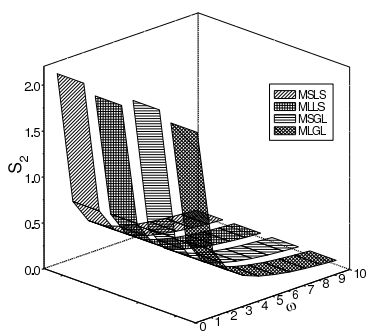

Figure 17: Variations of phase velocity $S_{2}$ with respect to frequency $\omega$ 


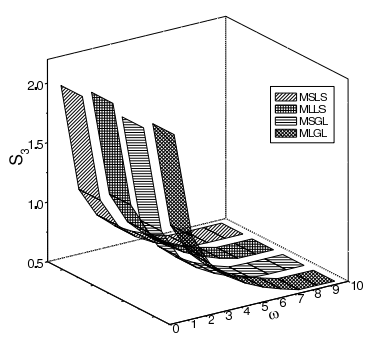

Figure 18: Variations of phase velocity $S_{3}$ with respect to frequency $\omega$

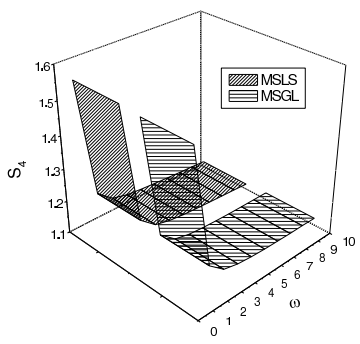

Figure 19: Variations of phase velocity $S_{4}$ with respect to frequency $\omega$ 


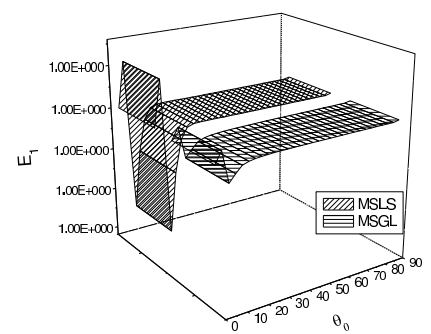

Figure 20: Variations of energy ratio $E_{1}$ with respect to angle of incidence $\theta_{0}$

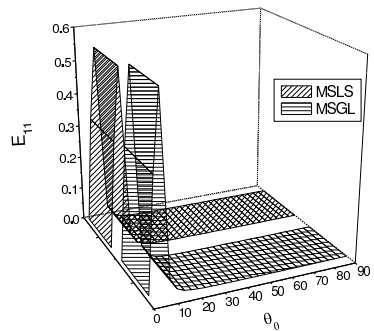

Figure 21: Variations of energy ratio $E_{11}$ with respect to angle of incidence $\theta_{0}$ 


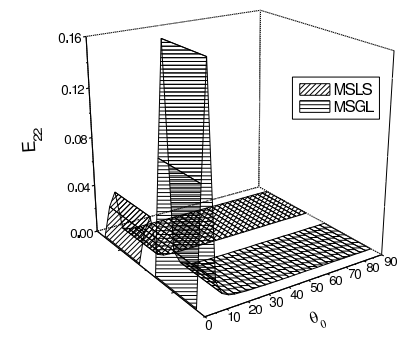

Figure 22: Variations of energy ratio $E_{22}$ with respect to angle of incidence $\theta_{0}$

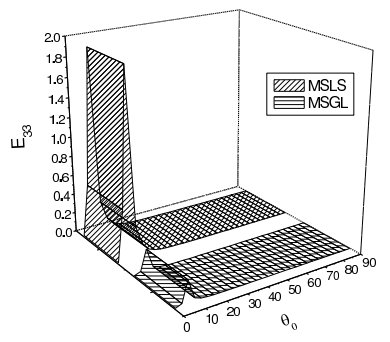

Figure 23: Variations of energy ratio $E_{33}$ with respect to angle of incidence $\theta_{0}$ 


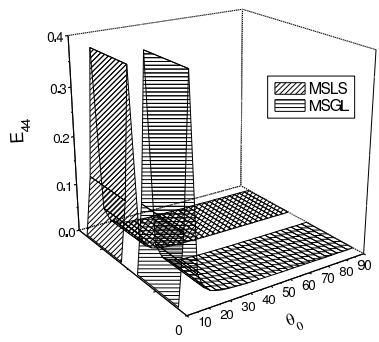

Figure 24: Variations of energy ratio $E_{44}$ with respect to angle of incidence $\theta_{0}$

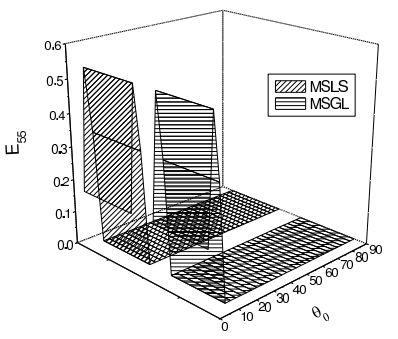

Figure 25: Variations of energy ratio $E_{55}$ with respect to angle of incidence $\theta_{0}$ 


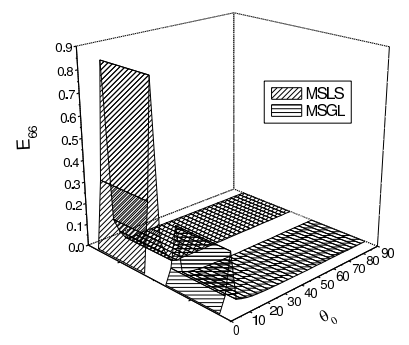

Figure 26: Variations of energy ratio $E_{66}$ with respect to angle of incidence $\theta_{0}$

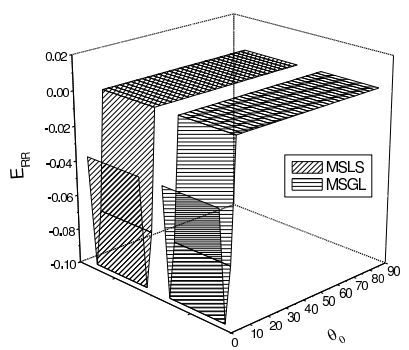

Figure 27: Variations of energy ratio $E_{R R}$ with respect to angle of incidence $\theta_{0}$ 


\section{References}

[1] Lord, H.W. and Shulman, Y., A generalized dynamical theory of thermoelasticity. Journal of Mechanics and Physics of Solids 15, 299-309, 1967.

[2] A.E. Green and K.A. Lindsay, Thermoelasticity, J. Elasticity, 2(1972) $1-7$.

[3] A. C. Eringen, Mechanics of micromorphic materials, in: Gortler, H. (ed.), Proceedings of the 2nd International Congress of Applied Mechanics, Springer, Berlin (1966) 131-138.

[4] A. C. Eringen, Mechanics of micromorphic continua, in: Kroner, E. (ed.), Mechanics of Generalized Continua, IUTAM Symposium, FreudenstadtStuttgart, Springer, Berlin (1968) 18-35.

[5] A. C. Eringen, Micropolar elastic solids with stretch, in: Prof. Dr. Mustaafa Inan Anisiana, Ari Kitabevi Matbassi 24 (1971) 1-18.

[6] A. C. Eringen, Theory of thermo-microstretch elastic solids, Int. J. Eng. Sci. 28, (1990) 1291-1301.

[7] A.C. Eringen, Microcontinuum Field Theories I: Foundations and Solids,Springer-Verlag, New York, (1999).

[8] M. Ciarletta and A. Scalia, Some results in linear theory of thermomicrostretch elastic solids, Meccanica 39(2004) 191-206.

[9] D. Iesan and R. Quintanilla, Thermal stresses in microstretch elastic plates, Int. J. Eng. Sci. 43, (2005) 885-907.

[10] M.I.A. Othman, K.H. Lotfy and R.M. Farouk, Generalized thermomicrostretch elastic medium with temperature dependent properties for different theories, Engineering Analysis with boundary elements 43 (2010) 229-237.

[11] F. Passarella and V. Tibullo, Some results in linear theory of thermoelasticity backward in time for microstretch materials, J. Thermal Stresses 33 (2010) 559-576.

[12] M. Marin, Lagrange identity method in thermoelasticity of bodies with microstructure, International Journal of Engineering Science, vol. 32, issue 8, (1994), 1229-1240.

[13] M. Marin, Some estimates on vibrations in thermoelasticity of dipolar bodies, Journal of Vibration and Control, vol.16 issue 1, (2010), 3347. 
[14] S. Kumar, J.N. Sharma and Y.D. Sharma, Generalized thermoelastic waves in microstretch platesloaded with fluid of varying temperature, Int. J. of Appl. Mechanics 3 (2011) 563-586.

[15] M.I.A. Othman and K.H. Lotfy, On the plane waves of generalized thermomicrostretch elastic half-space under three theories, Int. Commu. in Heat and Mass Transfer 37 (2010) 192-200.

[16] M.I.A. Othman and K.H. Lotfy, Effect of rotation of plane waves in generalized thermomicrostretch elastic solids with one relaxation time, Multi. Model. in Mat. and Str. 7 (2011) 43-62.

[17] R. Kumar and Rupender, Reflection at the free surface of magnetothermo-microstretch elastic solid, Bulletin of Polish Academy of Sciences 56 (2008) 263-271.

[18] R. Kumar and Rupender, Propagation of plane waves a imperfect boundary of elastic and electro-microstretch generalized thermoelastic solids, Appl. Math. and Mech. 30 (2012) 1445-1454.

[19] B. Singh, Reflection and refraction of plane waves at a liquid/thermomicrostretch elastic solid interface, Int. J. of Engg. Sci. 39(2001) 583-598.

[20] R. Kumar and G. Pratap, Refection of plane waves in a heat flux dependent microstretch thermoelastic solid half space, Int. J. App. Mech. and Engg. 10(2005) 253-266.

[21] J.N. Sharma, S. Kumar, and Y.D. Sharma, Propagation of Rayleigh waves in microstretch thermoelastic continua under inviscid fluid loadings, J. Thermal Stresses, 31(2008) 18-39.

[22] R. Kumar and G. Pratap, Wave propagation in microstretch thermoelaastic plate bordered with layers of inviscid liquid, Multi. Model. in Mat. Str., 5(2009) 171-184.

[23] W. Nowacki, Dynamical problems of thermodiffusion in solids-I, Bulletin of Polish Academy of Sciences Series, Science and Technology 22(1974(a)) $55-64$.

[24] W. Nowacki, Dynamical problems of thermodiffusion in solids-II, Bulletin of Polish Academy of Sciences Series, Science and Technology 22(1974(b)) 129-135.

[25] W. Nowacki, Dynamical problems of thermodiffusion in solids-III, Bulletin of Polish Academy of Sciences Series, Science and Technology 22(1974(c)) $275-276$. 
[26] W. Nowacki, Dynamical problems of diffusion in solids, Engineering Fracture Mechanics 8 (1976) 261-266.

[27] W. Dudziak and S.J. Kowalski, Theory of thermodiffusion for solids, Int. J. of Heat and Mass transfer 32(1989) 2005-2013.

[28] Z.S. Olesiak and Y.A. Pyryev, A coupled quasi-stationary problem of thermodiffusion for an elastic cylinder, Int. J. of Engg. Sci. 33(1995) 773-780.

[29] J.A. Gawinecki and A. Szymaniec, Global solution of the Cauchy problem in nonlinear thermoelastic diffusion in solid body, Proceedings in Appl. Math. and Mech., 1(2002) 446-447.

[30] H.H. Sherief, F.A. Hamza, and H.A. Saleh, The theory of generalized thermoelastic diffusion, International J. of Engg Sci., 42(2004) 591-608.

[31] M. Aouadi, Uniqueness and reciprocity theorems in the theory of generalized thermoelastic diffusion, J. Thermal Stresses, 30(2007) 665-678.

[32] H.H. Sherief, and H.A. Saleh, A half space problem in the theory of generalized thermoelastic diffusion, Int. J. of Solids and Structures, 42(2005) 4484-4493.

[33] Miglani, A. and Kaushal, S., Propagation of transverse and microrotational waves in micropolar generalized thermodiffusion elastic half space, ROMAI J., 7(2011) 125-139.

[34] R. Kumar and T. Kansal, Propagation of Lamb waves in transversely isotropic thermoelastic diffusive plate, Int. J. of Solids and Str., 45(2008) 5890-5913.

[35] R. Kumar and T. Kansal, Fundamental solution in the theory of thermomicrostretch elastic diffusive solids, International Scholarly Research Network, vol. 2011, Article ID 764632, 15 pages, doi:10.5402/2011/764632.

[36] R. Kumar, S.K. Garg and S. Ahuja, Propagation of plane waves at the interface of an elastic solid half-space and a microstretch thermoelastic diffusion solid half-space, Latin Amer. J. of Solids and Str., 10(2013) 1081-1108.

[37] R. Kumar, S.K. Garg and S. Ahuja, Rayleigh waves in isotropic microstrech thermoelastic diffusion solid half-space, Latin Amer. J. of Solids and Str., 111(2014) 299 - 319. 
[38] H. Kolsky, Stress waves in solids, Clarendon Press, Oxford, Dover press New York, 1963.

[39] J.D. Achenbach, Wave propagation in elastic solids, North-Holland, Amsterdam, 1973.

[40] R.D. Borcherdt, Reflection-refraction of general $P$ and type-I $S$ waves in elastic and anelastic solids, Geophysical Journal of Royal Astronomical Society, 70(1982) 621-638.

[41] A. C. Eringen, Plane waves in non local micropolar elasticity, Int. J. Eng. Sci. 22(1984) 1113-1121.

[42] M. Marin, G. Stan, Weak solutions in Elasticity of dipolar bodies with stretch, Carpathian Journal of Mathematics , Vol. 29 (1), 2013, pp. 33-40

[43] K. Sharma, M. Marin, Reflection and transmission of waves from imperfect boundary between two heat conducting micropolar thermoelastic solids, An. Sti. Univ. Ovidius Constanta, Vol. 22, issue 2,(2014), 151-175

[44] M. Marin, O. Florea, On temporal behavior of solutions in Thermoelasticity of porous micropolar bodies, An. Sti. Univ. Ovidius Constanta, Vol. 22 , issue $1,(2014), 169-188$

[45] X. Lin, B. Zhao and Z. Du, A third-order multi-point boundary value problem at resonance with one three dimensional kernel space, Carpathian Journal of Mathematics, Vol. 30 (2014), No. 1, 93-100

Rajneesh KUMAR Department of Mathematics,

Kurukshetra University,

Kurukshetra-136 119, India

E-mail: rajneesh_kuk@rediffmail.com, 
WAVE PROPAGATION IN A MICROSTRETCH THERMOELASTIC DIFFUSION SOLID 